\title{
Adakitic rocks associated with the Shilu copper-molybdenum deposit in the Yangchun Basin, South China, and their tectonic implications
}

\author{
Lipeng Zhang ${ }^{1,2} \cdot{\text { Yongbin } \mathrm{Hu}^{1} \cdot \text { Jinlong Liang }}^{3}$ - Trevor Ireland ${ }^{4}$. \\ Youliang Chen ${ }^{3}$ Rongqing Zhang ${ }^{1}$ Saijun Sun ${ }^{1}$ Weidong Sun ${ }^{5,6}$
}

Received: 14 December 2016/Revised: 15 January 2017/Accepted: 6 February 2017/Published online: 17 February 2017

(C) The Author(s) 2017. This article is published with open access at Springerlink.com

\begin{abstract}
South China is famous for the extensive magmatism and polymetallic mineralization that took place there in the Mesozoic. Shilu is a large porphyry-skarn $\mathrm{Cu}-\mathrm{Mo}$ deposit in the Yangchun Basin, South China. The lithology of the Shilu intrusion is granodiorite and quartz diorite, both of which are high-K calc-alkaline series, with high $\mathrm{Sr}$ (>400 ppm) content along with low $\mathrm{Y}$ and $\mathrm{Yb}$ contents. Most of the samples have characteristics of adakite except for a few samples that have slightly higher $\mathrm{Y}$ and $\mathrm{Yb}$ contents, which may be plausibly explained by crustal contamination. Laser Ablation Inductively Coupled Plasma Mass Spectrometry zircon $\mathrm{U}-\mathrm{Pb}$ dating revealed ages between $106.6 \pm 1.3$ and $103.9 \pm 0.5 \mathrm{Ma}$, with multiple magmatic pulses. Molybdenite Re-Os isochron age of $102.2 \pm 2.9 \mathrm{Ma}$
\end{abstract}

Electronic supplementary material The online version of this article (doi:10.1007/s11631-017-0146-6) contains supplementary material, which is available to authorized users.

Weidong Sun

weidongsun@gig.ac.cn

1 CAS Key Laboratory of Mineralogy and Metallogeny, Guangzhou Institute of Geochemistry, Chinese Academy of Sciences, Guangzhou 510640, China

2 University of Chinese Academy of Sciences, Beijing 100094, China

3 Department of Geochemistry, Chengdu University of Technology, Chengdu 610059, China

4 Research School of Earth Sciences, The Australian National University, Bldg 61 Mills Road Acton ACT, Canberra, ACT 0200, Australia

5 Center of Deep Sea Research, Institute of Oceanography, Chinese Academy of Sciences, Qingdao 266071, China

6 CAS Center for Excellence in Tibetan Plateau Earth Sciences, Chinese Academy of Science, Beijing 100101, China
(MSWD $=9.4)$ was determined, which is identical to the youngest zircon $\mathrm{U}-\mathrm{Pb}$ age $(103.9 \pm 0.5 \mathrm{Ma})$ within error. The Shilu intrusion has high oxygen fugacity as indicated by high zircon $\mathrm{Ce}^{4+} / \mathrm{Ce}^{3+}$ and $\mathrm{Eu}_{\mathrm{N}} / \mathrm{Eu}_{\mathrm{N}} *$ ratios. Considering the geochemical characteristics (high $\mathrm{Sr}$, and low $\mathrm{Y}$ and $\mathrm{Yb}$ contents), high oxygen fugacity, and copper mineralization of the Shilu intrusion, it was most likely formed by partial melting of a subducted young oceanic slab. Whole-rock $\mathrm{Sr}-$ $\mathrm{Nd}$ isotope-, zircon $\mathrm{Hf}$ isotope-, and whole-rock trace element analyses show that Shilu adakitic magmas may have interacted with type II enriched mantle and/or crustal materials during ascent. South China was affected by the Pacific tectonic regime to the east and the Neo-Tethys tectonic regime to the south in the Cretaceous. Based on the Pacific Plate drifting and rotation history, it is hard to explain how the Pacific Plate would have subducted and melted, forming adakitic rocks in the Shilu region. Considering the tectonic history of Southeast Asia and the South China Sea, the Neo-Tethys trench should have been much closer to the South China Block in the Cretaceous, and thus have had a greater impact on the South China Block. Based on the subduction direction, time of subduction, and distance between the Neo-Tethys subduction zone and the Shilu deposit, subduction of the Neo-Tethys ridge is the best mechanism for explaining the Shilu adakitic rocks and $\mathrm{Cu}-$ Mo mineralization.

Keywords Shilu deposit - Adakitic rocks - Cretaceous · Neo-Tethys · South China

\section{Introduction}

In the Cretaceous, South China was surrounded by several continental terranes and oceanic plates, such as the North China Block to the north, the Songpan-Ganzi Block to the 
west, the Indochina Block to the southwest, the Pacific Plate to the east, and the Neo-Tethys Plate to the south. South China is famous for large-scale magmatism and related mineralization, especially in the Mesozoic. A variety of tectonic models have been proposed to explain these characteristics specifically and the Mesozoic tectonic evolution of South China more generally (Gilder et al. 1991, 1996; Li 2000; Zhou and Li 2000; Zhou et al. 2006; Li et al. 2007, 2012; Sun et al. 2007a, 2012b; Ling et al. 2009; Mao et al. 2011; Wang et al. 2011). The models can be classified into three groups: active continental margin related to subduction of the Pacific Plate; continental rifting and extension; and mantle plume event. More recently, it has been proposed that the Neo-Tethys Plate subducted beneath the South China Block in the Cretaceous (Sun et al. 2016b; Sun 2016).

In addition to the giant $\mathrm{W}, \mathrm{Sn}, \mathrm{Sb}, \mathrm{Nb}, \mathrm{Ta}, \mathrm{U}$, and rareearth element (REE) deposit belts, a number of copper deposits occur in South China as well. Porphyry or skarn copper deposits are the most important deposit types, accounting for $\sim 70 \%-80 \%$ of the world's total copper reserves (Sillitoe 2010; Sun et al. 2015a), and mainly form along convergent belts; for example: the Andean region in South America. Copper deposits in the northeast part of the South China Block have been well-studied. In contrast, the genesis and tectonic setting of copper deposits in the southern part of the South China Block remain obscure.

Shilu is a porphyry-skarn $\mathrm{Cu}-\mathrm{Mo}$ deposit in the Yangchun Basin, in the south of the South China Block. Previous studies on this deposit have considered geologic features as well as geochronology, geochemistry, and mineral trace element composition. A range of ages have been obtained through different techniques, including a zircon U-Pb evaporation age of $125 \mathrm{Ma}$, a mineral $\mathrm{Rb}-\mathrm{Sr}$ age of $122 \mathrm{Ma}$, an ${ }^{40} \mathrm{Ar}-{ }^{39} \mathrm{Ar}$ age of $\sim 100 \mathrm{Ma}$, and orebearing and ore-barren granodiorite Laser Ablation Inductively Coupled Plasma Mass Spectrometry (LA-ICPMS) zircon U-Pb ages of $\sim 107$ and $\sim 104-107 \mathrm{Ma}$, respectively (Zhao et al. 1985; Yu et al. 1998; Duan et al. 2013; Zheng et al. 2015). Therefore, the formation age of the Shilu intrusion remains controversial. For the oreforming age, a molybdenite Re-Os isochron age of $104.1 \pm 1.3$ Ma has been obtained (Zhao et al. 2012). The genesis and tectonic setting of the deposit are also ambiguous. It is located close to the transition zone between the eastern Tethys and the Pacific tectonic realms. The effect of the two major tectonic realms on this region remains unclear. Some previous work has proposed that the Shilu intrusion formed in an extensional environment related to subduction of the Pacific Plate (Duan et al. 2013; Zheng et al. 2015). In addition, the variation of magma compositions has been used to argue that the region belonged to an intraplate setting and experienced upwelling
Fig. 1 a Sketch geologic map of South China and surrounding plates. b Geological map of the Yangchun basin, South China. c Geological map of the Shilu $\mathrm{Cu}-\mathrm{Mo}$ deposit. The black pentacles are sample sites

of the asthenosphere mantle and extension of overlying lithosphere during the Early Jurassic to the Early Cretaceous ( $\mathrm{Li}$ et al. 2001).

In this study, we present LA-ICP-MS zircon U-Pb ages, molybdenite $\mathrm{Re}-\mathrm{Os}$ ages, zircon Hf isotopes, and wholerock major and trace elements. These data provide firmer constraints on the timing and geochemical characteristics of the Shilu intrusion and deposit, with implications for the tectonic setting of Late Cretaceous magmas in this region.

\section{Geological setting}

\subsection{Regional geology}

The Shilu $\mathrm{Cu}-\mathrm{Mo}$ deposit is located in the Yangchun Basin, South China (Fig. 1). The Yangchun Basin is a NESW-trending fault-bounded basin formed from an Indosinian synclinorium, southeast of the Wuchuan-Sihui deep fault (Li et al. 2000; Zheng et al. 2015). The area has complex geological structure and has experienced intensive magmatic activity. NE-SW-trending faults define the tectonic framework. E-W-trending faults can also be found, e.g., the Taishan-Yangchun tectonic belt (Zhang 2008).

The outcropping strata in this basin include Cambrian, Devonian, Carboniferous, Jurassic, and Quaternary strata (Fig. 1; Luo 1988; Zheng et al. 2013). The basement is composed of Cambrian low-grade metamorphic flysch and sandy shales distributed on both sides of the basin, which are covered by Upper Paleozoic carbonate rocks, neritic clastic rocks, and sandy shales. Jurassic terrestrial clastic rocks unconformably overlie the Paleozoic strata (Zheng et al. 2013).

Abundant granitic intrusions of different rock types with varying mineralization have been recognized. These intrusions vary from intermediate to acidic in composition, including monzonite, monzonitic granite, granodiorite, and granite (Fig. 1). The ages of these rocks range from Caledonian to Yanshanian, with most magmatic activity having occurred in the Yanshanian. Several polymetallic deposits closely related to granitic intrusions have been found, such as the Shilu porphyry-skarn $\mathrm{Cu}-\mathrm{Mo}$ deposit, the BengkengShiwu $\mathrm{Cu}-\mathrm{Pb}-\mathrm{Zn}$ deposit, the Xishan $\mathrm{Sn}-\mathrm{W}$ deposit, and the Yingwuling W-Sn deposit (Chen 1988; Wu and Zeng 2011; Duan et al. 2013; Mei et al. 2013; Zheng et al. 2015).

\subsection{Ore deposit geology}

Shilu is a porphyry-skarn $\mathrm{Cu}-\mathrm{Mo}$ deposit with average grades of $0.9 \%$ for $\mathrm{Cu}$ and $0.21 \%$ for Mo (Zheng et al. 


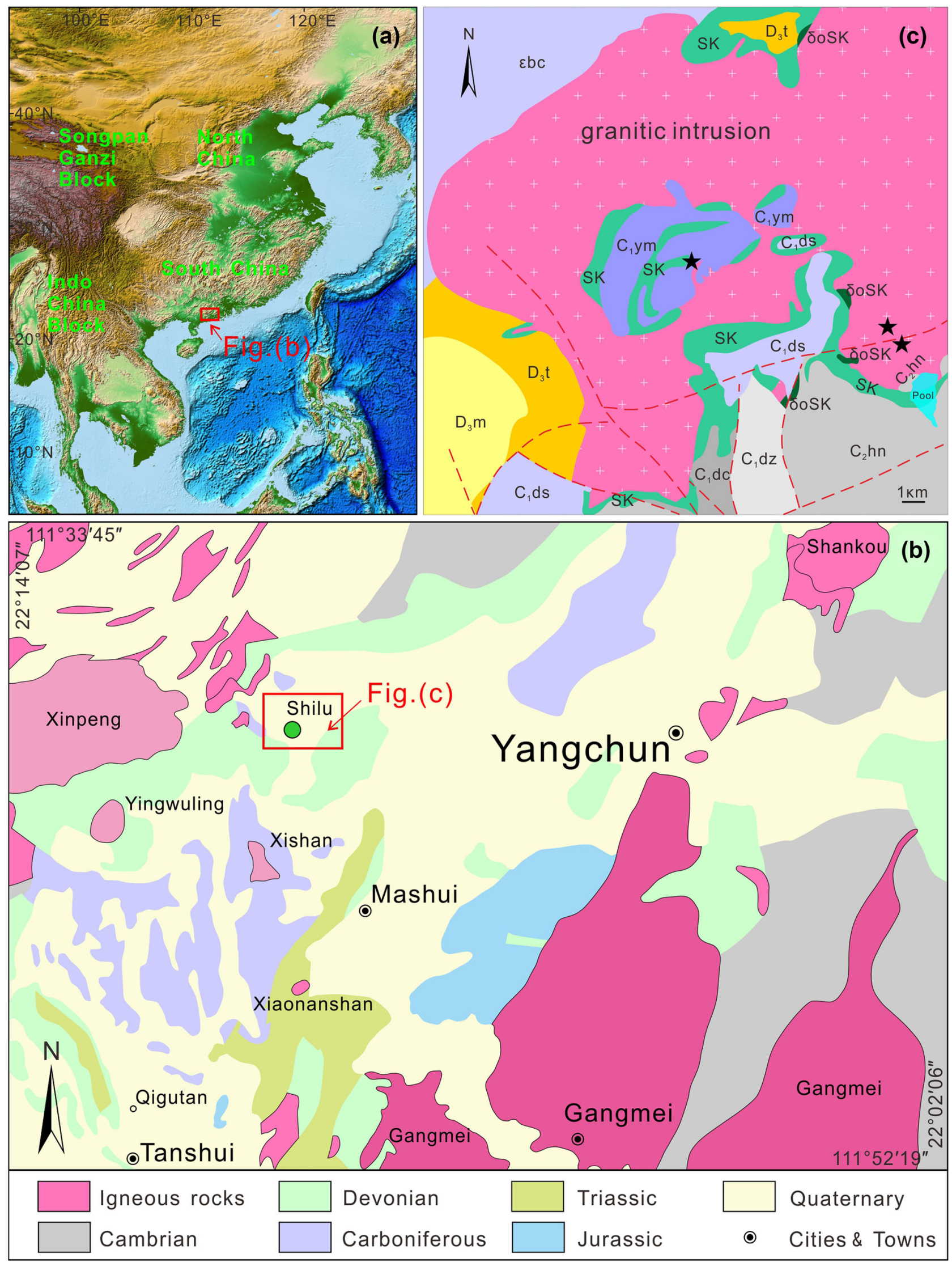


2015). NE-trending faults are the dominant tectonic framework in this mine, in keeping with the regional fault structure. Ore bodies can be divided into two ore types: oxidized ore and primary ore. The oxidized ore bodies are mainly distributed in weathered skarn and Quaternary strata, and were an important ore type in the past. The primary ore bodies are mainly hosted in the skarn belts between intrusions and carbonate country rocks (Fig. 1; Zhang 2008; Zhang and Zhang 2012).

The strata cropping out in the deposit include the Cambrian Bacun Group (cbc); the Upper Devonian Maozifeng Formation $\left(\mathrm{D}_{3} \mathrm{~m}\right)$ and Tianziling Formation $\left(\mathrm{D}_{3} \mathrm{t}\right)$; the Lower Carboniferous Menggongao Formation $\left(\mathrm{C}_{1} \mathrm{ym}\right)$ sandstone and shale; the Shidengzi Segment $\left(\mathrm{C}_{1} \mathrm{ds}\right)$ limestone; the Ceshui Segment $\left(\mathrm{C}_{1} \mathrm{dc}\right)$ sandstone, shale, and hornstone; the Zimenqiao Segment $\left(\mathrm{C}_{1} \mathrm{dz}\right)$ dolomite and limestone; the Middle Carboniferous Huanglongqun Group $\left(\mathrm{C}_{2} \mathrm{hn}\right)$ dolomite and limestone; and Quaternary sediments (Fig. 1).

Different petrographic features were recognized for the Shilu intrusion based on field observation and thin section studies under optical microscope. Granodiorite and quartz diorite are the main rock types (Fig. 2a, b). The granodiorite samples were composed of plagioclase $(\sim 60 \%)$, K-feldspar $(\sim 15 \%)$, quartz $(\sim 20 \%)$, biotite $(\sim 8 \%)$, and minor amphibole $(<5 \%)$ (Fig. 2e). More dark minerals and sulfides were observed in the quartz diorite as compared to granodiorite (Fig. 2e, f). The quartz diorite samples primarily consisted of plagioclase $(\sim 55 \%)$, K-feldspar $(\sim 12 \%)$, quartz $(\sim 18 \%)$, biotite $(\sim 8 \%)$, and amphibole $(\sim 8 \%)$ (Fig. $2 \mathrm{f})$. Both granodiorite and quartz diorite samples contained accessory minerals of magnetite, zircon, apatite, and titanite.

\section{Samples and analytical methods}

\subsection{Whole-rock major and trace elements}

Nine fresh intrusive rock samples, derived from outcrops and drill holes, were collected from different positions in the deposit (Fig. 1). They were broken to small fragments, washed and dried, and then crushed to 200 mesh. Wholerock analyses for major and trace elements were conducted at the ALS Laboratory Group, an Australian analytical laboratory in China. The major elements were analyzed using X-ray fluorescence spectrometry (XRF), specifically a PANalytical Axios. First, the loss on ignition (LOI) values were determined. Samples were weighed and then heated in a muffle furnace and then weighed after ignition to calculate the LOI. Then, the baked whole-rock powders were mixed with lithium borate and fused at over $1000{ }^{\circ} \mathrm{C}$ in a smelting furnace. The whole-rock major elements were then measured using XRF. To analyze trace elements, fused samples were progressively dissolved in nitric acid, hydrochloric acid, and hydrofluoric acid, and then analyzed by an Agilent $7700 \times$ ICP-MS. Whole-rock F content was analyzed by ion electrodes using an American Accumet AR50. Whole-rock $\mathrm{Cl}$ content was determined by ion chromatography on a Switzerland Metrohm IC930.

\subsection{Zircon U-Pb dating}

Three samples used for zircon $\mathrm{U}-\mathrm{Pb}$ dating were collected from surface and drill-hole localities (Fig. 1c). Zircon grains were separated from the samples using standard density and magnetic separation techniques. The separated grains were fixed in epoxy and then polished down to near half section to expose internal structures. Cathodoluminescence and optical microscopy images were taken of zircon grains, and were used for choosing appropriate spots for laser ablation. Zircon $\mathrm{U}-\mathrm{Pb}$ ages and trace element compositions were analyzed using LA-ICP-MS at the Key Laboratory of Mineralogy and Metallogeny, Guangzhou Institute of Geochemistry, Chinese Academy of Sciences. The LA-ICP-MS system is comprised of a Resonetics RESOlution S-155 ArF-Excimer laser source $(\lambda=193 \mathrm{~nm})$ and an Agilent 7900 ICP-MS. The ablation conditions were $4 \mathrm{~J} \cdot \mathrm{cm}^{-2}$ laser energy, ablation frequency of $8 \mathrm{~Hz}$, and a spot diameter of $29 \mu \mathrm{m}$. The SRM 610 glass and the TEMORA zircon were used as external calibration standards and the ${ }^{91} \mathrm{Zr}$ was selected as the internal standard. Detailed information for analytical procedures and conditions are described by Li et al. (2012). Data processing was performed using ICPMSDataCal (Liu et al. 2008; Lin et al. 2016). Concordia diagrams and weighted mean age calculations were processed using Isoplot (Ludwig 2003).

\subsection{Molybdenite Re-Os dating}

Four molybdenite samples were selected for Re-Os isochron dating. Samples SL27-1 and SL33 were derived from quartz veins with metal mineralization, and samples ZK4503-662 and ZK4503-686 were from molybdenite veins in skarn. Samples were purified using the alcohol floating method and then molybdenite grains were carefully chosen under binocular microscope, before being crushed to 200 mesh in an agate mortar. Re-Os isotope analyses were conducted at the State Key Laboratory of Isotope Geochemistry, Guangzhou Institute of Geochemistry, Chinese Academy of Sciences, using Thermo Scientific X Series 2 ICP-MS. Detailed information for analytical conditions and procedures can be obtained from earlier publications (Sun et al. 2010b, 2015b). 
Fig. 2 Specimen photographs and micrographs of the Shilu intrusion. a Granodiorite; b Quartz diorite; c Molybdenite, chalcopyrite and pyrite in quartz vein; d Skarn; e Micrograph of granodiorite, cross-polarized light; f Micrograph of quartz diorite, cross-polarized light. Am amphibole, $B t$ biotite, $M t$ magnetite, $P l$ plagioclase, $Q z$ quartz
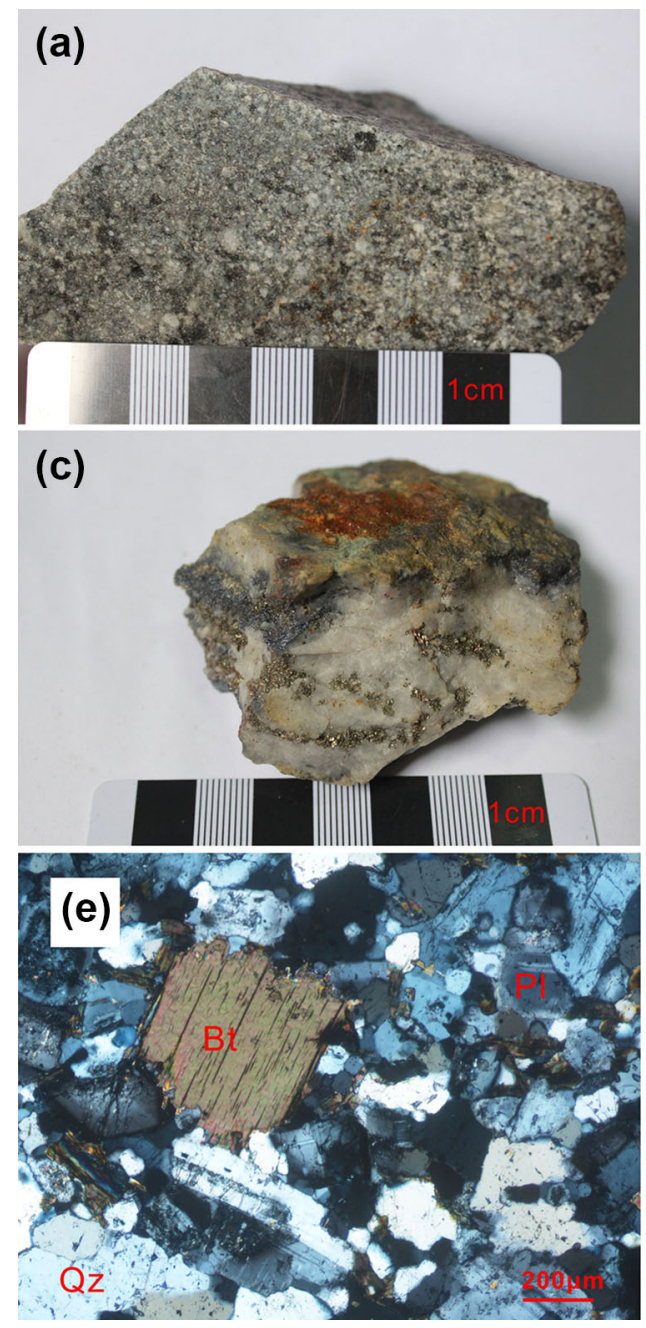

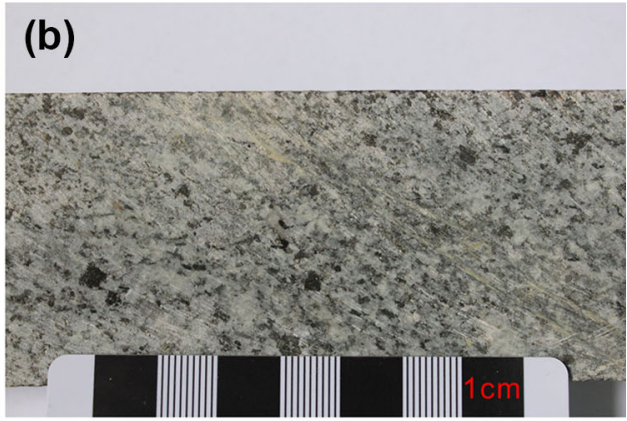

(d)
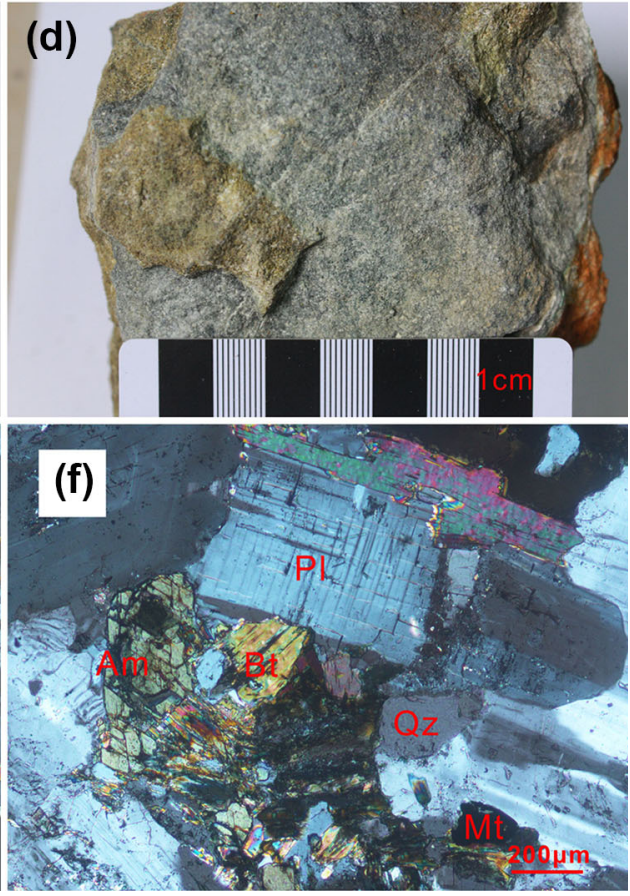

\subsection{Zircon Hf isotope analyses}

In-situ zircon Hf isotope analyses were performed on a Resonetics M-50 laser ablation system coupled with a Neptune MC-ICP-MS at the State Key Laboratory of Isotope Geochemistry, Guangzhou Institute of Geochemistry, Chinese Academy of Sciences. A spot diameter of $45 \mu \mathrm{m}$ and an ablation frequency of $8 \mathrm{~Hz}$ were used for analyzing the spots where $\mathrm{U}-\mathrm{Pb}$ had been analyzed. The zircon Penglai was used as a reference standard, with a recommended ${ }^{176} \mathrm{Hf} /{ }^{177} \mathrm{Hf}$ ratio of $0.282906 \pm 0.000001(2 \mathrm{~s})(\mathrm{Li}$ et al. 2010). Detailed analytical methods have been described by Wu et al. (2006).

\section{Results}

\subsection{Whole-rock major and trace elements}

Major and trace element data are presented in Table 1. Our data are plotted in Fig. 3, along with previous results from other studies. Most of the samples have LOI values less than 2 $\mathrm{wt} \%$. The major element compositions vary slightly, with $\mathrm{SiO}_{2}$ contents of $64.5 \mathrm{wt} \%-67.4 \mathrm{wt} \%, \mathrm{Al}_{2} \mathrm{O}_{3}$ contents of $15.4 \mathrm{wt} \%-16.6 \mathrm{wt} \%, \mathrm{MgO}$ contents of $1.66 \mathrm{wt} \%-2.52 \mathrm{wt} \%$, $\mathrm{Na}_{2} \mathrm{O}$ contents of $2.43 \mathrm{wt} \%-2.99 \mathrm{wt} \%$, and $\mathrm{K}_{2} \mathrm{O}$ contents of $2.68 \mathrm{wt} \%-3.56 \mathrm{wt} \%$. In the $\mathrm{SiO}_{2}$ against $\mathrm{K}_{2} \mathrm{O}$ diagram, the Shilu intrusion belongs to the high-K calc-alkaline series (Fig. 3a). The diagram of A/CNK versus A/NK shows that the intrusive rocks are metaluminous $(\mathrm{A} / \mathrm{CNK}<1.1)$ (Fig. 3b). Volatile contents show low fluorine (520-590 ppm) and high chlorine (110-220 ppm) characteristics.

All the samples, including previously published data, show similar chondrite-normalized REE patterns (Fig. 4a), with enrichment of LREE [LREE/HREE $=7.69-11.0$, $\left.(\mathrm{La} / \mathrm{Yb})_{\mathrm{N}}=7.78-13.4\right]$, and slightly negative Eu anomalies $(\delta \mathrm{Eu}=0.75-0.82)$. In the primitive mantle normalized spidergram (Fig. 4b), all samples display characteristics of arc magmatic rocks, with positive anomalies of $\mathrm{U}, \mathrm{K}$, and $\mathrm{Pb}$ and negative anomalies of $\mathrm{Nb}, \mathrm{Ta}, \mathrm{P}$, and $\mathrm{Ti}$ for high field-strength elements. In addition, the Shilu pluton has high $\mathrm{Sr}$ and low $\mathrm{Y}$ and $\mathrm{Yb}$ contents, indicating the absence 
Table 1 The compositions of major oxides (wt\%) and trace elements (ppm) for the Shilu intrusion

\begin{tabular}{|c|c|c|c|c|c|c|c|c|c|}
\hline Lithology & $\begin{array}{l}\text { SL01-1 } \\
\text { Granodiorite }\end{array}$ & Granodiorite & $\begin{array}{l}\text { SL01-3 } \\
\text { Granodiorite }\end{array}$ & $\begin{array}{l}\text { SL30 } \\
\text { Quartz } \\
\text { diorite }\end{array}$ & $\begin{array}{l}\text { ZK8502- } \\
481 \\
\text { Quartz } \\
\text { diorite }\end{array}$ & $\begin{array}{l}\text { ZK8502- } \\
486 \\
\text { Quartz } \\
\text { diorite }\end{array}$ & $\begin{array}{l}\text { ZK8502- } \\
509 \\
\text { Quartz } \\
\text { diorite }\end{array}$ & $\begin{array}{l}\text { ZK8502- } \\
514 \\
\text { Quartz } \\
\text { diorite }\end{array}$ & $\begin{array}{l}\text { ZK8502- } \\
594 \\
\text { Quartz } \\
\text { diorite }\end{array}$ \\
\hline $\mathrm{SiO}_{2}$ & 65.6 & 66.7 & 67.4 & 64.6 & 64.8 & 64.5 & 64.7 & 64.8 & 65.6 \\
\hline $\mathrm{TiO}_{2}$ & 0.42 & 0.40 & 0.38 & 0.47 & 0.48 & 0.48 & 0.48 & 0.43 & 0.41 \\
\hline $\mathrm{Al}_{2} \mathrm{O}_{3}$ & 16.6 & 16.3 & 15.9 & 16.2 & 15.4 & 15.8 & 15.7 & 15.9 & 15.9 \\
\hline $\mathrm{Fe}_{2} \mathrm{O}_{3}$ & 3.31 & 3.33 & 3.07 & 2.40 & 4.51 & 4.62 & 4.54 & 4.11 & 3.99 \\
\hline $\mathrm{MnO}$ & 0.05 & 0.04 & 0.04 & 0.05 & 0.09 & 0.09 & 0.09 & 0.07 & 0.08 \\
\hline $\mathrm{MgO}$ & 1.85 & 1.74 & 1.66 & 2.52 & 2.21 & 2.22 & 2.17 & 2.01 & 1.93 \\
\hline $\mathrm{CaO}$ & 4.22 & 4.33 & 4.16 & 4.96 & 4.59 & 4.75 & 5.05 & 5.34 & 5.07 \\
\hline $\mathrm{Na}_{2} \mathrm{O}$ & 2.98 & 2.99 & 2.90 & 2.43 & 2.66 & 2.89 & 2.83 & 2.97 & 2.96 \\
\hline $\mathrm{K}_{2} \mathrm{O}$ & 3.11 & 3.00 & 2.89 & 3.28 & 3.56 & 3.03 & 2.93 & 2.68 & 2.80 \\
\hline $\mathrm{P}_{2} \mathrm{O}_{5}$ & 0.21 & 0.20 & 0.18 & 0.18 & 0.21 & 0.18 & 0.19 & 0.19 & 0.19 \\
\hline LOI & 1.33 & 0.90 & 1.07 & 2.53 & 0.95 & 1.17 & 1.22 & 1.35 & 0.94 \\
\hline Total & 99.85 & 100.07 & 99.76 & 99.77 & 99.6 & 99.87 & 99.93 & 99.95 & 99.94 \\
\hline $\mathrm{Na}_{2} \mathrm{O}+\mathrm{K}_{2} \mathrm{O}$ & 6.09 & 5.99 & 5.79 & 5.71 & 6.22 & 5.92 & 5.76 & 5.65 & 5.76 \\
\hline $\mathrm{K}_{2} \mathrm{O} / \mathrm{Na}_{2} \mathrm{O}$ & 1.04 & 1.00 & 1.00 & 1.35 & 1.34 & 1.05 & 1.04 & 0.90 & 0.95 \\
\hline $\mathrm{A} / \mathrm{NK}$ & 2.01 & 1.99 & 2.01 & 2.14 & 1.87 & 1.97 & 2.00 & 2.04 & 2.01 \\
\hline $\mathrm{A} / \mathrm{CNK}$ & 1.04 & 1.01 & 1.03 & 0.97 & 0.93 & 0.95 & 0.92 & 0.91 & 0.93 \\
\hline V & 73 & 74 & 69 & 89 & 92 & 100 & 97 & 90 & 88 \\
\hline $\mathrm{Cr}$ & 20 & 20 & 20 & 20 & 30 & 30 & 30 & 30 & 30 \\
\hline $\mathrm{Ga}$ & 18.7 & 18.1 & 17.6 & 16.5 & 15.2 & 16.1 & 16.2 & 15.6 & 15.9 \\
\hline $\mathrm{Rb}$ & 85.9 & 77.7 & 79.3 & 86.7 & 111 & 115 & 119 & 113 & 113 \\
\hline $\mathrm{Sr}$ & 581 & 584 & 579 & 583 & 439 & 462 & 443 & 450 & 454 \\
\hline $\mathrm{Y}$ & 19.5 & 18.0 & 15.9 & 18.9 & 22.3 & 21.6 & 21.0 & 19.9 & 21.2 \\
\hline $\mathrm{Zr}$ & 177 & 174 & 157 & 144 & 183 & 137 & 149 & 149 & 142 \\
\hline $\mathrm{Nb}$ & 7.9 & 7.5 & 7.0 & 8.2 & 8.6 & 9.2 & 8.7 & 7.9 & 8.0 \\
\hline Cs & 3.07 & 2.49 & 2.66 & 4.46 & 3.89 & 4.71 & 5.08 & 5.37 & 6.73 \\
\hline $\mathrm{Ba}$ & 825 & 786 & 738 & 690 & 856 & 542 & 585 & 510 & 532 \\
\hline $\mathrm{La}$ & 30.5 & 30.9 & 28.9 & 27.5 & 27.5 & 26.8 & 29.8 & 25.0 & 24.4 \\
\hline $\mathrm{Ce}$ & 61.0 & 62.0 & 56.6 & 57.3 & 54.5 & 52.6 & 58.5 & 49.5 & 49.6 \\
\hline $\operatorname{Pr}$ & 6.94 & 6.94 & 6.30 & 6.51 & 6.27 & 5.91 & 6.46 & 5.51 & 5.64 \\
\hline $\mathrm{Nd}$ & 26.6 & 26.0 & 22.9 & 23.9 & 23.4 & 22.0 & 23.5 & 21.0 & 21.1 \\
\hline $\mathrm{Sm}$ & 5.15 & 4.70 & 4.37 & 4.58 & 4.45 & 4.30 & 4.51 & 4.37 & 4.22 \\
\hline $\mathrm{Eu}$ & 1.10 & 1.10 & 1.03 & 1.14 & 1.13 & 1.04 & 1.15 & 1.05 & 1.04 \\
\hline Gd & 3.92 & 3.71 & 3.38 & 3.90 & 4.41 & 3.80 & 4.21 & 4.03 & 3.92 \\
\hline $\mathrm{Tb}$ & 0.59 & 0.59 & 0.48 & 0.58 & 0.69 & 0.64 & 0.64 & 0.58 & 0.66 \\
\hline Dy & 3.39 & 3.18 & 3.00 & 3.42 & 4.17 & 3.63 & 3.84 & 3.54 & 3.78 \\
\hline Но & 0.68 & 0.62 & 0.58 & 0.66 & 0.84 & 0.77 & 0.75 & 0.73 & 0.78 \\
\hline $\mathrm{Er}$ & 1.92 & 1.82 & 1.70 & 1.84 & 2.27 & 2.21 & 2.25 & 1.98 & 2.13 \\
\hline $\mathrm{Tm}$ & 0.27 & 0.30 & 0.24 & 0.29 & 0.33 & 0.33 & 0.32 & 0.32 & 0.34 \\
\hline $\mathrm{Yb}$ & 1.91 & 1.89 & 1.55 & 1.80 & 2.30 & 2.05 & 2.04 & 2.08 & 2.25 \\
\hline $\mathrm{Lu}$ & 0.28 & 0.27 & 0.25 & 0.29 & 0.35 & 0.37 & 0.32 & 0.33 & 0.34 \\
\hline Hf & 4.9 & 4.7 & 4.3 & 3.9 & 4.8 & 3.8 & 4.0 & 4.0 & 3.8 \\
\hline Ta & 0.6 & 0.5 & 0.5 & 0.5 & 0.9 & 0.9 & 0.9 & 0.7 & 0.9 \\
\hline W & 2 & 2 & 2 & 4 & 5 & 3 & 20 & 10 & 4 \\
\hline Th & 12.2 & 11.5 & 10.7 & 9.4 & 11.1 & 10.6 & 11.2 & 8.91 & 11.4 \\
\hline $\mathrm{U}$ & 3.30 & 3.16 & 2.71 & 3.25 & 3.85 & 4.87 & 3.88 & 4.29 & 5.97 \\
\hline F & 590 & & & 590 & & & 570 & & 520 \\
\hline
\end{tabular}


Table 1 continued

\begin{tabular}{|c|c|c|c|c|c|c|c|c|c|}
\hline Samples & SL01-1 & SL01-2 & SL01-3 & SL30 & $\begin{array}{l}\text { ZK8502- } \\
481\end{array}$ & $\begin{array}{l}\text { ZK8502- } \\
486\end{array}$ & $\begin{array}{l}\text { ZK8502- } \\
509\end{array}$ & $\begin{array}{l}\text { ZK8502- } \\
514\end{array}$ & $\begin{array}{l}\text { ZK8502- } \\
594\end{array}$ \\
\hline Lithology & Granodiorite & Granodiorite & Granodiorite & $\begin{array}{l}\text { Quartz } \\
\text { diorite }\end{array}$ & $\begin{array}{l}\text { Quartz } \\
\text { diorite }\end{array}$ & $\begin{array}{l}\text { Quartz } \\
\text { diorite }\end{array}$ & $\begin{array}{l}\text { Quartz } \\
\text { diorite }\end{array}$ & $\begin{array}{l}\text { Quartz } \\
\text { diorite }\end{array}$ & $\begin{array}{l}\text { Quartz } \\
\text { diorite }\end{array}$ \\
\hline $\mathrm{Cl}$ & 160 & & & 110 & & & 220 & & 190 \\
\hline LREE & 135 & 135 & 123 & 124 & 121 & 116 & 127 & 110 & 109 \\
\hline HREE & 13.0 & 12.4 & 11.2 & 12.8 & 15.4 & 13.8 & 14.4 & 13.6 & 14.2 \\
\hline$\sum \mathrm{REE}$ & 148 & 148 & 135 & 137 & 136 & 130 & 142 & 123 & 123 \\
\hline LREE/HREE & 10.4 & 10.9 & 11.0 & 9.73 & 7.85 & 8.40 & 8.86 & 8.08 & 7.69 \\
\hline$\delta \mathrm{Eu}$ & 0.75 & 0.81 & 0.82 & 0.82 & 0.78 & 0.79 & 0.81 & 0.76 & 0.78 \\
\hline $\mathrm{Sr} / \mathrm{Y}$ & 29.8 & 32.4 & 36.4 & 30.9 & 19.7 & 21.4 & 21.1 & 22.6 & 21.4 \\
\hline$(\mathrm{La} / \mathrm{Yb})_{\mathrm{N}}$ & 11.5 & 11.7 & 13.4 & 11.0 & 8.58 & 9.38 & 10.5 & 8.62 & 7.78 \\
\hline
\end{tabular}

$\delta \mathrm{Eu}=\mathrm{Eu}_{\mathrm{N}} /\left(\mathrm{Sm}_{\mathrm{N}} * \mathrm{Gd}_{\mathrm{N}}\right)^{1 / 2}$. The Subscript $\mathrm{N}$ represents chondrite-normalized, and chondrite data are from Sun and McDonough (1989)
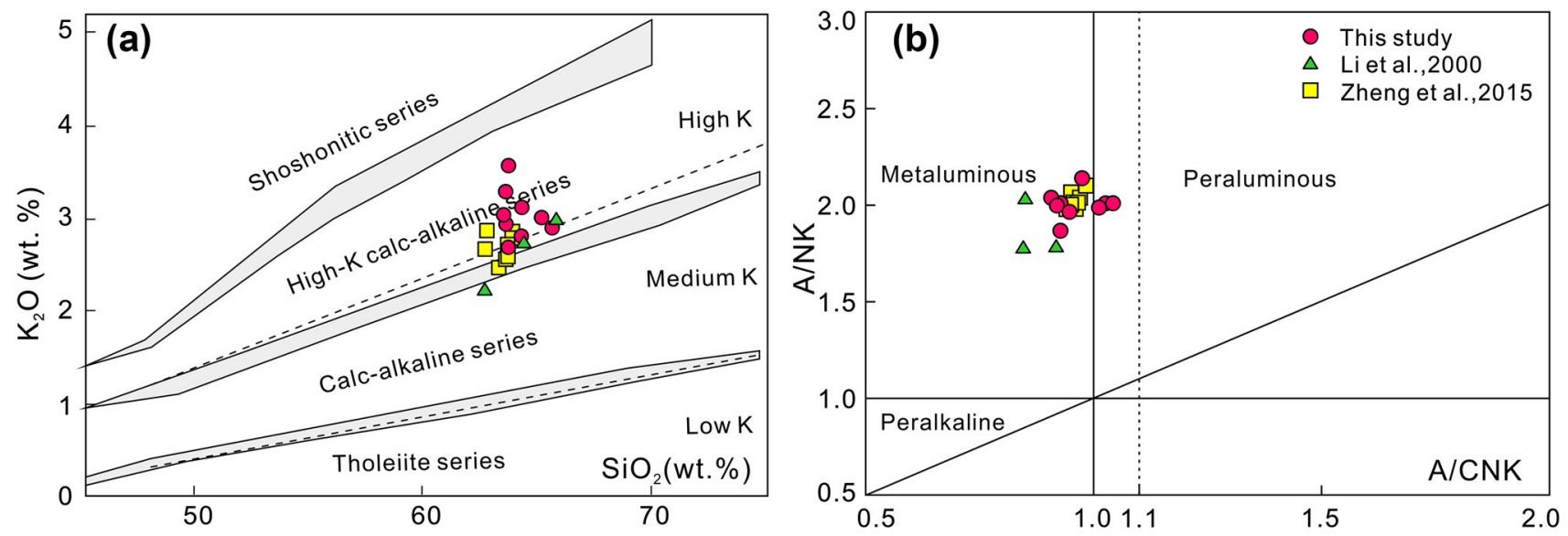

Fig. 3 a The diagram of $\mathrm{SiO}_{2}$ versus $\mathrm{K}_{2} \mathrm{O}$ and $\mathbf{b}$ the diagram of $\mathrm{A} / \mathrm{CNK}$ versus $\mathrm{A} / \mathrm{NK}$ for the Shilu intrusion

of plagioclase (both residual and crystallized) and residual garnet in the magma source. However, some previous data (Zheng et al. 2015) have shown strong $\mathrm{Zr}$ and Hf negative anomalies, which have resulted from incomplete dissolution of the zircon samples during analysis.

\subsection{Zircon U-Pb dating and trace elements}

Three samples from the Shilu intrusion, including granodiorite and quartz diorite, were selected for zircon $\mathrm{U}-\mathrm{Pb}$ dating. The results of isotopic compositions and ages are listed in Table 2. We obtained 23, 17, and 39 valid data points for samples SL01, SL30, and ZK8502-594, respectively, according to the following three criteria: (1) no cracks, defects, or inclusions; (2) more than $30 \mathrm{~s}$ of laser ablation time; and (3) a highly concordant age. Most zircon grains exhibited a euhedral prismatic shape with lengths of 100-300 $\mu \mathrm{m}$. As in the CL image presented, zircon grains showed typical oscillatory zones (Fig. 5). The analytical results show that all zircon grains had high $\mathrm{Th} / \mathrm{U}$ ratios (average value $0.43,0.55$, and 0.54 , respectively), typical of magmatic zircon (Sun et al. 2002; Wu and Zheng 2004). The $\mathrm{U}-\mathrm{Pb}$ concordia diagrams for the three samples are shown in Fig. 5. The weighted mean ${ }^{206} \mathrm{~Pb} /{ }^{238} \mathrm{U}$ ages for SL01, SL30, and ZK8502-594 were $106.6 \pm 1.3,104.4 \pm 1.0$, and $103.9 \pm 0.5 \mathrm{Ma}$, respectively (Fig. 5). Different zircon ages between granodiorite and quartz diorite indicate multiple magmatic pulses.

Zircon trace element data were obtained along with zircon U-Pb analyses. These are listed in Supplementary material Table $\mathrm{S} 1$ together with zircon $\mathrm{Ce}^{4+} / \mathrm{Ce}^{3+}$ ratios, and $\mathrm{Eu}_{\mathrm{N}} / \mathrm{Eu}_{\mathrm{N}}{ }^{*}$ ratios. As shown in the chondrite-normalized REE patterns (Fig. 6), all zircon grains presented typical patterns, i.e. enriched in HREE and depleted in LREE. They exhibited slightly negative Eu and strongly positive $\mathrm{Ce}$ anomalies.

\subsection{Molybdenite Re-Os dating}

Molybdenite is a frequently-used mineral for determining ore-forming age, because it is a common mineral in a 

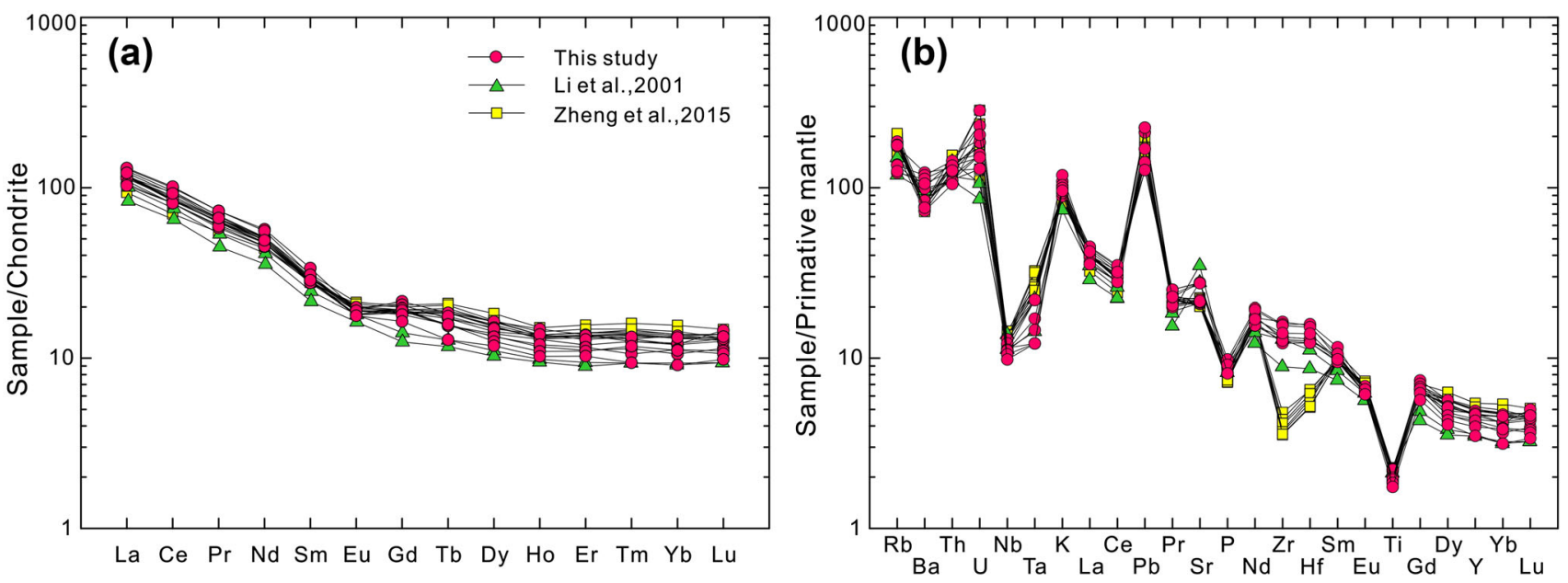

Fig. 4 a Chondrite-normalized rare earth elements pattern and $\mathbf{b}$ primitive mantle-normalized incompatible elements spider diagram for the Shilu intrusion. Chondrite and primitive mantle data are from Sun and McDonough (1989)

variety of deposits and its osmium content is mainly radiogenic due to low common osmium and high rhenium concentrations. Re-Os isotopic compositions of four molybdenite samples are listed in Table 3. Four samples had variable concentrations of ${ }^{187} \mathrm{Re}$ and ${ }^{187} \mathrm{Os}$, ranging from 2.43 to $201.24 \mathrm{ppm}$ and 4.11 to $343.90 \mathrm{ppb}$, respectively. Molybdenite Re-Os model ages were calculated using the formula: $\mathrm{t}=(1 / \lambda) * \ln \left(1+{ }^{187} \mathrm{Os} /{ }^{187} \mathrm{Re}\right)$, where $\lambda$ is the decay constant of ${ }^{187} \operatorname{Re}\left(1.666 \times 10^{-11}\right.$ year $\left.^{-1}\right)$ (Smoliar et al. 1996). The model ages for samples SL27-1, SL33, ZK4503-662, and ZK4503-686 were $101.3 \pm$ $0.4 \mathrm{Ma}(2 \sigma), 102.5 \pm 0.4 \mathrm{Ma}(2 \sigma), 101.5 \pm 0.9 \mathrm{Ma}(2 \sigma)$, and $101.7 \pm 0.6 \mathrm{Ma}(2 \sigma)$, respectively. All the samples had identical model ages within error, yielding a weighted mean age of $101.9 \pm 1.0 \mathrm{Ma} \quad(\mathrm{MSWD}=6.6)$. The ${ }^{187} \mathrm{Re}-{ }^{187} \mathrm{Os}$ isochron age was $102.2 \pm 2.9 \mathrm{Ma}$ (MSWD = 9.4) (Fig. 7), which is consistent with the weighted mean of the model ages within error. The large MSWD is likely due to variation of initial Os content or formation age.

\subsection{Zircon Hf isotopes}

Zircon Hf isotope compositions are listed in Supplementary material Table S2. Twenty-two zircon grains were analyzed from SL01 and twenty grains from ZK8502-594. Most ${ }^{176} \mathrm{Lu} /{ }^{177} \mathrm{Hf}$ ratios were less than 0.002 , indicating there has been little radiogenic ${ }^{176} \mathrm{Hf}$ accumulation since zircon crystallization. The initial ${ }^{176} \mathrm{Hf} /{ }^{177} \mathrm{Hf}$ ratios were calculated using the measured ${ }^{176} \mathrm{Lu} /{ }^{177} \mathrm{Hf}$ ratios and the ${ }^{176} \mathrm{Lu}$ decay constant of $1.865 \times 10^{-11}$ year $^{-1}$ (Scherer et al. 2001). The zircon $\varepsilon_{\mathrm{Hf}}(\mathrm{t})$ values ranged from -10.7 to -3.3 , and were calculated using the corresponding zircon age, the chondritic ${ }^{176} \mathrm{Hf} /{ }^{177} \mathrm{Hf}$ ratio of 0.282772 , and ${ }^{176} \mathrm{Lu} /{ }^{177} \mathrm{Hf}$ ratio of 0.0332 (BlichertToft and Albarede 1997).

\section{Discussion}

\subsection{Oxygen fugacity of magma}

Oxygen fugacity is an important factor for porphyry copper deposits (Mungall 2002; Sillitoe 2010; Sun et al. 2013b, 2015a). Zircon is a good recorder of magmatic oxygen fugacity (Ballard et al. 2002). Ce and Eu have $\mathrm{Ce}^{3+}, \mathrm{Ce}^{4+}$ and $\mathrm{Eu}^{2+}, \mathrm{Eu}^{3+}$ valence states, respectively, which have different partition coefficients in zircon. $\mathrm{Ce}^{4+}$ and $\mathrm{Eu}^{3+}$ are more easily accommodated in zircon than $\mathrm{Ce}^{3+}$ and $\mathrm{Eu}^{2+}$, respectively. Therefore, $\mathrm{Ce}^{4+} / \mathrm{Ce}^{3+}$ and $\mathrm{Eu}_{\mathrm{N}} / \mathrm{Eu}_{\mathrm{N}} *$ ratios in zircon can reflect relative oxidation states of magmas (Ballard et al. 2002; Liang et al. 2006). Ballard et al. (2002) proposed that porphyry copper mineralization is closely associated with zircon $\mathrm{Ce}^{4+}$ / $\mathrm{Ce}^{3+}>300$ and $\mathrm{Eu}_{\mathrm{N}} / \mathrm{Eu}_{\mathrm{N}} *>0.4$, which are useful indices for evaluating the mineralization potential. In this study, the zircon $\mathrm{Ce}^{4+} / \mathrm{Ce}^{3+}$ ratios of Shilu ranged from 138 to 1114 , with an average value of $\sim 619$; and the $\mathrm{Eu}_{\mathrm{N}} / \mathrm{Eu}_{\mathrm{N}}{ }^{*}$ ratios ranged from 0.43 to 0.59 , with an average value of $\sim 0.53$. Both indicators are significantly higher than the reference values. In the $\mathrm{Eu}_{\mathrm{N}} / \mathrm{Eu}_{\mathrm{N}} *$ versus $\mathrm{Ce}^{4+} / \mathrm{Ce}^{3+}$ diagram (Fig. 8), most of the Shilu samples plotted in the overlap fields of ore-bearing porphyry in Chuquicamata-El Abra and Dexing porphyry copper deposits (Zhang et al. 2013), indicating that the Shilu intrusion crystallized from highly oxidized magma. The chemical composition of biotite in the granodiorite also displayed a high oxygen fugacity between nickel-nickel oxide and the hematitemagnetite buffer (Zheng et al. 2015). Magmas of porphyry or skarn copper deposits generally have high oxygen fugacity, usually more than two orders of magnitude higher than the fayalite-magnetite-quartz $(\Delta \mathrm{FMQ}+2)$ oxygen buffer (Mungall 2002), but lower than the hematite- 
Table 2 LA-ICP-MS zircon U-Pb dating results for the Shilu intrusion

\begin{tabular}{|c|c|c|c|c|c|c|c|c|c|c|c|c|c|}
\hline \multirow[t]{2}{*}{ Spot no. } & \multirow{2}{*}{$\begin{array}{l}\mathrm{Pb} \\
\mathrm{ppm}\end{array}$} & \multirow{2}{*}{$\begin{array}{l}\text { Th } \\
\text { ppm }\end{array}$} & \multirow{2}{*}{$\begin{array}{l}\mathrm{U} \\
\mathrm{ppm}\end{array}$} & \multirow[t]{2}{*}{$\mathrm{Th} / \mathrm{U}$} & \multicolumn{2}{|c|}{${ }^{207} \mathrm{~Pb} /{ }^{235} \mathrm{U}$} & \multicolumn{2}{|c|}{${ }^{206} \mathrm{~Pb} /{ }^{238} \mathrm{U}$} & \multicolumn{2}{|l|}{${ }^{207} \mathrm{~Pb} /{ }^{235} \mathrm{U}$} & \multicolumn{2}{|l|}{${ }^{206} \mathrm{~Pb} /{ }^{238} \mathrm{U}$} & \multirow[t]{2}{*}{ Concordance } \\
\hline & & & & & Ratio & $1 \sigma$ & Ratio & $1 \sigma$ & Age (Ma) & $1 \sigma$ & Age (Ma) & $1 \sigma$ & \\
\hline \multicolumn{14}{|l|}{ SL01 } \\
\hline 1 & 11.07 & 227 & 577 & 0.39 & 0.1059 & 0.0049 & 0.01626 & 0.00027 & 102.2 & 4.5 & 104.0 & 1.7 & $98 \%$ \\
\hline 2 & 16.13 & 325 & 791 & 0.41 & 0.1149 & 0.0047 & 0.0167 & 0.00022 & 110.5 & 4.2 & 106.8 & 1.4 & $96 \%$ \\
\hline 3 & 6.14 & 131 & 303 & 0.43 & 0.1187 & 0.0070 & 0.01662 & 0.00028 & 113.9 & 6.4 & 106.2 & 1.8 & $93 \%$ \\
\hline 4 & 15.13 & 454 & 696 & 0.65 & 0.1073 & 0.0045 & 0.01677 & 0.00028 & 103.5 & 4.1 & 107.2 & 1.8 & $96 \%$ \\
\hline 5 & 11.37 & 216 & 551 & 0.39 & 0.115 & 0.0055 & 0.01725 & 0.00030 & 110.5 & 5.0 & 110.3 & 1.9 & $99 \%$ \\
\hline 6 & 9.01 & 212 & 450 & 0.47 & 0.111 & 0.0060 & 0.01618 & 0.00025 & 106.9 & 5.4 & 103.5 & 1.6 & $96 \%$ \\
\hline 7 & 5.97 & 115 & 311 & 0.37 & 0.1035 & 0.0069 & 0.01569 & 0.00028 & 100.0 & 6.4 & 100.4 & 1.8 & $99 \%$ \\
\hline 8 & 6.25 & 115 & 318 & 0.36 & 0.1058 & 0.0070 & 0.01613 & 0.00028 & 102.1 & 6.4 & 103.2 & 1.8 & $98 \%$ \\
\hline 9 & 9.47 & 254 & 458 & 0.55 & 0.1161 & 0.0068 & 0.01599 & 0.00025 & 111.5 & 6.2 & 102.3 & 1.6 & $91 \%$ \\
\hline 10 & 8.5 & 178 & 434 & 0.41 & 0.1119 & 0.0076 & 0.01682 & 0.00029 & 107.7 & 6.9 & 107.5 & 1.8 & $99 \%$ \\
\hline 11 & 11.16 & 181 & 553 & 0.33 & 0.1201 & 0.0063 & 0.01692 & 0.00023 & 115.2 & 5.7 & 108.2 & 1.5 & $93 \%$ \\
\hline 12 & 14.29 & 297 & 719 & 0.41 & 0.1069 & 0.0063 & 0.01664 & 0.00023 & 103.1 & 5.7 & 106.4 & 1.5 & $96 \%$ \\
\hline 13 & 5.22 & 72 & 256 & 0.28 & 0.1113 & 0.0086 & 0.01749 & 0.00036 & 107.2 & 7.9 & 111.7 & 2.3 & $95 \%$ \\
\hline 14 & 7.6 & 152 & 387 & 0.39 & 0.1157 & 0.0071 & 0.01615 & 0.00028 & 111.2 & 6.4 & 103.3 & 1.8 & $92 \%$ \\
\hline 15 & 5.83 & 128 & 290 & 0.44 & 0.1035 & 0.0068 & 0.01652 & 0.00029 & 100.0 & 6.2 & 105.6 & 1.8 & $94 \%$ \\
\hline 16 & 6.41 & 141 & 306 & 0.46 & 0.1214 & 0.0080 & 0.01731 & 0.00034 & 116.3 & 7.3 & 110.6 & 2.1 & $94 \%$ \\
\hline 17 & 9.36 & 188 & 456 & 0.41 & 0.1173 & 0.0070 & 0.01698 & 0.00030 & 112.7 & 6.4 & 108.6 & 1.9 & $96 \%$ \\
\hline 18 & 7.59 & 151 & 363 & 0.42 & 0.1123 & 0.0081 & 0.01749 & 0.00035 & 108.1 & 7.4 & 111.8 & 2.2 & $96 \%$ \\
\hline 19 & 15.4 & 320 & 723 & 0.44 & 0.1198 & 0.0060 & 0.01732 & 0.00029 & 114.9 & 5.4 & 110.7 & 1.8 & $96 \%$ \\
\hline 20 & 9.69 & 239 & 455 & 0.53 & 0.1065 & 0.0061 & 0.01664 & 0.00026 & 102.8 & 5.6 & 106.4 & 1.6 & $96 \%$ \\
\hline 21 & 6.21 & 122 & 289 & 0.42 & 0.1131 & 0.0086 & 0.01724 & 0.00035 & 108.8 & 7.9 & 110.2 & 2.2 & $98 \%$ \\
\hline 22 & 14.98 & 232 & 698 & 0.33 & 0.1158 & 0.0060 & 0.01699 & 0.00025 & 111.3 & 5.5 & 108.6 & 1.6 & $97 \%$ \\
\hline 23 & 6.4 & 143 & 293 & 0.49 & 0.109 & 0.0082 & 0.01678 & 0.00036 & 105 & 7.5 & 107.3 & 2.3 & $97 \%$ \\
\hline \multicolumn{14}{|l|}{ SL30 } \\
\hline 1 & 6.99 & 193 & 312 & 0.62 & 0.1108 & 0.0051 & 0.01666 & 0.00030 & 106.7 & 4.6 & 106.5 & 1.9 & $99 \%$ \\
\hline 2 & 6.81 & 144 & 339 & 0.42 & 0.1088 & 0.0054 & 0.01678 & 0.00026 & 104.9 & 5.0 & 107.3 & 1.6 & $97 \%$ \\
\hline 3 & 26.97 & 971 & 1194 & 0.81 & 0.1084 & 0.0030 & 0.01668 & 0.00017 & 104.5 & 2.8 & 106.7 & 1.1 & $97 \%$ \\
\hline 4 & 10.85 & 240 & 552 & 0.44 & 0.1126 & 0.0039 & 0.01601 & 0.00019 & 108.4 & 3.6 & 102.4 & 1.2 & $94 \%$ \\
\hline 5 & 5.69 & 109 & 282 & 0.38 & 0.119 & 0.0053 & 0.01683 & 0.00025 & 114.2 & 4.8 & 107.6 & 1.6 & $94 \%$ \\
\hline 6 & 12.14 & 373 & 590 & 0.63 & 0.1086 & 0.0037 & 0.01603 & 0.00020 & 104.7 & 3.4 & 102.5 & 1.3 & $97 \%$ \\
\hline 7 & 6.03 & 184 & 303 & 0.61 & 0.0939 & 0.0048 & 0.01602 & 0.00024 & 91.1 & 4.5 & 102.4 & 1.5 & $88 \%$ \\
\hline 8 & 12.68 & 366 & 597 & 0.61 & 0.1145 & 0.0046 & 0.01657 & 0.00023 & 110.1 & 4.2 & 105.9 & 1.4 & $96 \%$ \\
\hline 9 & 12.6 & 395 & 606 & 0.65 & 0.1127 & 0.0047 & 0.01607 & 0.00029 & 108.4 & 4.3 & 102.8 & 1.8 & $94 \%$ \\
\hline 10 & 8.66 & 194 & 434 & 0.45 & 0.1114 & 0.0057 & 0.0162 & 0.00023 & 107.3 & 5.2 & 103.6 & 1.5 & $96 \%$ \\
\hline 11 & 13.57 & 268 & 687 & 0.39 & 0.1156 & 0.0055 & 0.01645 & 0.00026 & 111.1 & 5.0 & 105.2 & 1.7 & $94 \%$ \\
\hline 12 & 15.64 & 367 & 810 & 0.45 & 0.1126 & 0.0048 & 0.01603 & 0.00026 & 108.3 & 4.4 & 102.5 & 1.6 & $94 \%$ \\
\hline 13 & 8.13 & 159 & 415 & 0.38 & 0.111 & 0.0053 & 0.01647 & 0.00024 & 106.9 & 4.8 & 105.3 & 1.5 & $98 \%$ \\
\hline 14 & 16.59 & 593 & 780 & 0.76 & 0.1056 & 0.0037 & 0.01629 & 0.00021 & 102.0 & 3.4 & 104.2 & 1.3 & $97 \%$ \\
\hline 15 & 13.04 & 459 & 619 & 0.74 & 0.1049 & 0.0038 & 0.01606 & 0.00023 & 101.3 & 3.5 & 102.7 & 1.4 & $98 \%$ \\
\hline 16 & 7.83 & 172 & 405 & 0.42 & 0.1125 & 0.0050 & 0.01595 & 0.00022 & 108.2 & 4.6 & 102.0 & 1.4 & $94 \%$ \\
\hline 17 & 11.46 & 293 & 560 & 0.52 & 0.1149 & 0.0042 & 0.01661 & 0.00023 & 110.4 & 3.8 & 106.2 & 1.5 & $96 \%$ \\
\hline \multicolumn{14}{|c|}{ ZK8502-594 } \\
\hline 1 & 13.26 & 428 & 656 & 0.65 & 0.1095 & 0.0051 & 0.01667 & 0.00025 & 105.5 & 4.7 & 106.6 & 1.6 & $99 \%$ \\
\hline 2 & 9.41 & 260 & 465 & 0.56 & 0.1041 & 0.0045 & 0.01654 & 0.00022 & 100.6 & 4.1 & 105.8 & 1.4 & $94 \%$ \\
\hline 3 & 10.65 & 330 & 527 & 0.63 & 0.1082 & 0.0040 & 0.01601 & 0.00022 & 104.3 & 3.7 & 102.4 & 1.4 & $98 \%$ \\
\hline 4 & 8.56 & 226 & 431 & 0.53 & 0.1056 & 0.0053 & 0.01629 & 0.00025 & 101.9 & 4.9 & 104.2 & 1.6 & $97 \%$ \\
\hline
\end{tabular}


Table 2 continued

\begin{tabular}{|c|c|c|c|c|c|c|c|c|c|c|c|c|c|}
\hline \multirow[t]{2}{*}{ Spot no. } & \multirow{2}{*}{$\begin{array}{l}\mathrm{Pb} \\
\mathrm{ppm}\end{array}$} & \multirow{2}{*}{$\begin{array}{l}\text { Th } \\
\text { ppm }\end{array}$} & \multirow{2}{*}{$\begin{array}{l}\mathrm{U} \\
\mathrm{ppm}\end{array}$} & \multirow[t]{2}{*}{$\mathrm{Th} / \mathrm{U}$} & \multicolumn{2}{|c|}{${ }^{207} \mathrm{~Pb} /{ }^{235} \mathrm{U}$} & \multicolumn{2}{|c|}{${ }^{206} \mathrm{~Pb} /{ }^{238} \mathrm{U}$} & \multicolumn{2}{|l|}{${ }^{207} \mathrm{~Pb} /{ }^{235} \mathrm{U}$} & \multicolumn{2}{|l|}{${ }^{206} \mathrm{~Pb} /{ }^{238} \mathrm{U}$} & \multirow[t]{2}{*}{ Concordance } \\
\hline & & & & & Ratio & $1 \sigma$ & Ratio & $1 \sigma$ & Age (Ma) & $1 \sigma$ & Age (Ma) & $1 \sigma$ & \\
\hline 5 & 7.73 & 185 & 394 & 0.47 & 0.1013 & 0.0043 & 0.01624 & 0.00021 & 98.0 & 3.9 & 103.9 & 1.3 & $94 \%$ \\
\hline 6 & 14.29 & 497 & 697 & 0.71 & 0.1074 & 0.0038 & 0.01607 & 0.00022 & 103.6 & 3.5 & 102.8 & 1.4 & $99 \%$ \\
\hline 7 & 3.85 & 61 & 202 & 0.3 & 0.1101 & 0.0067 & 0.01667 & 0.00032 & 106.1 & 6.1 & 106.6 & 2 & $99 \%$ \\
\hline 8 & 7.06 & 149 & 357 & 0.42 & 0.1067 & 0.0058 & 0.01625 & 0.00022 & 102.9 & 5.4 & 103.9 & 1.4 & $99 \%$ \\
\hline 9 & 7.39 & 190 & 365 & 0.52 & 0.1087 & 0.0056 & 0.01648 & 0.00023 & 104.8 & 5.1 & 105.4 & 1.5 & $99 \%$ \\
\hline 10 & 10.94 & 339 & 536 & 0.63 & 0.1058 & 0.0040 & 0.01577 & 0.00022 & 102.1 & 3.6 & 100.8 & 1.4 & $98 \%$ \\
\hline 11 & 8.38 & 201 & 424 & 0.47 & 0.1199 & 0.0054 & 0.01592 & 0.00027 & 115.0 & 4.9 & 101.8 & 1.7 & $87 \%$ \\
\hline 12 & 7.65 & 189 & 379 & 0.5 & 0.1154 & 0.0060 & 0.01638 & 0.00024 & 110.9 & 5.4 & 104.8 & 1.5 & $94 \%$ \\
\hline 13 & 11.69 & 559 & 498 & 1.12 & 0.1307 & 0.0065 & 0.01661 & 0.00028 & 124.8 & 5.8 & 106.2 & 1.8 & $83 \%$ \\
\hline 14 & 9.74 & 340 & 467 & 0.73 & 0.1283 & 0.0061 & 0.01679 & 0.00029 & 122.6 & 5.5 & 107.3 & 1.8 & $86 \%$ \\
\hline 15 & 5.85 & 132 & 301 & 0.44 & 0.1068 & 0.0055 & 0.01619 & 0.00026 & 103.0 & 5.0 & 103.6 & 1.7 & $99 \%$ \\
\hline 16 & 5.56 & 145 & 283 & 0.51 & 0.1180 & 0.0058 & 0.01611 & 0.00026 & 113.3 & 5.3 & 103.1 & 1.7 & $90 \%$ \\
\hline 17 & 9.02 & 226 & 469 & 0.48 & 0.1089 & 0.0044 & 0.01612 & 0.00025 & 105.0 & 4.0 & 103.1 & 1.6 & $98 \%$ \\
\hline 18 & 9.95 & 316 & 500 & 0.63 & 0.1060 & 0.0043 & 0.01587 & 0.00021 & 102.3 & 4.0 & 101.5 & 1.3 & $99 \%$ \\
\hline 19 & 7.92 & 216 & 405 & 0.53 & 0.1102 & 0.0045 & 0.01615 & 0.00024 & 106.1 & 4.2 & 103.3 & 1.5 & $97 \%$ \\
\hline 20 & 18.54 & 458 & 932 & 0.49 & 0.1069 & 0.0040 & 0.01637 & 0.00021 & 103.1 & 3.6 & 104.7 & 1.4 & $98 \%$ \\
\hline 21 & 7.28 & 166 & 382 & 0.44 & 0.1031 & 0.0055 & 0.01627 & 0.00023 & 99.6 & 5.1 & 104.0 & 1.5 & $95 \%$ \\
\hline 22 & 11.37 & 361 & 543 & 0.66 & 0.1053 & 0.0044 & 0.01599 & 0.00021 & 101.7 & 4.0 & 102.3 & 1.4 & $99 \%$ \\
\hline 23 & 7.01 & 157 & 376 & 0.42 & 0.1055 & 0.0050 & 0.01602 & 0.00025 & 101.9 & 4.6 & 102.5 & 1.6 & $99 \%$ \\
\hline 24 & 11 & 356 & 549 & 0.65 & 0.1074 & 0.0047 & 0.01612 & 0.00025 & 103.6 & 4.3 & 103.1 & 1.6 & $99 \%$ \\
\hline 25 & 8.73 & 200 & 451 & 0.44 & 0.1148 & 0.0053 & 0.01653 & 0.00026 & 110.3 & 4.8 & 105.7 & 1.6 & $95 \%$ \\
\hline 26 & 4.69 & 104 & 239 & 0.44 & 0.1098 & 0.0073 & 0.01680 & 0.00032 & 105.7 & 6.7 & 107.4 & 2.0 & $98 \%$ \\
\hline 27 & 6.77 & 143 & 355 & 0.4 & 0.1183 & 0.0057 & 0.01620 & 0.00021 & 113.5 & 5.2 & 103.6 & 1.3 & $90 \%$ \\
\hline 28 & 8.21 & 176 & 424 & 0.42 & 0.1157 & 0.0054 & 0.01627 & 0.00024 & 111.2 & 4.9 & 104.0 & 1.5 & $93 \%$ \\
\hline 29 & 6.43 & 154 & 330 & 0.47 & 0.1098 & 0.0050 & 0.01598 & 0.00021 & 105.8 & 4.6 & 102.2 & 1.3 & $96 \%$ \\
\hline 30 & 9.12 & 229 & 468 & 0.49 & 0.1099 & 0.0042 & 0.01609 & 0.00022 & 105.8 & 3.9 & 102.9 & 1.4 & $97 \%$ \\
\hline 31 & 13.2 & 366 & 667 & 0.55 & 0.1076 & 0.0042 & 0.01636 & 0.00027 & 103.8 & 3.9 & 104.6 & 1.7 & $99 \%$ \\
\hline 32 & 9.89 & 258 & 503 & 0.51 & 0.1028 & 0.0044 & 0.01616 & 0.00022 & 99.4 & 4.1 & 103.3 & 1.4 & $96 \%$ \\
\hline 33 & 8.88 & 199 & 456 & 0.44 & 0.1060 & 0.0042 & 0.01599 & 0.00020 & 102.3 & 3.8 & 102.3 & 1.3 & $99 \%$ \\
\hline 34 & 12.45 & 387 & 606 & 0.64 & 0.1241 & 0.0049 & 0.01660 & 0.00023 & 118.8 & 4.4 & 106.1 & 1.5 & $88 \%$ \\
\hline 35 & 8.83 & 232 & 456 & 0.51 & 0.1054 & 0.0046 & 0.01623 & 0.00022 & 101.7 & 4.2 & 103.8 & 1.4 & $98 \%$ \\
\hline 36 & 6.3 & 151 & 323 & 0.47 & 0.1022 & 0.0054 & 0.01654 & 0.00026 & 98.8 & 5.0 & 105.8 & 1.7 & $93 \%$ \\
\hline 37 & 5.65 & 136 & 286 & 0.47 & 0.1078 & 0.0059 & 0.01623 & 0.00024 & 103.9 & 5.4 & 103.8 & 1.5 & $99 \%$ \\
\hline 38 & 8.8 & 249 & 434 & 0.57 & 0.1187 & 0.0052 & 0.01662 & 0.00025 & 113.9 & 4.7 & 106.3 & 1.6 & $93 \%$ \\
\hline 39 & 6.31 & 201 & 312 & 0.65 & 0.1067 & 0.0052 & 0.01632 & 0.00026 & 103.0 & 4.8 & 104.3 & 1.7 & $98 \%$ \\
\hline
\end{tabular}

magnetite oxygen buffer (Sun et al. 2013b, 2015a). Oxygen fugacity strongly affects the sulfur species in magmas and their solubility. Sulfur in magmas is mainly present as sulfate when the oxygen fugacity is higher than $\triangle \mathrm{FMQ}+2$, and the sulfur solubility in magmas increases from $\sim 1000 \mathrm{ppm}$ to $>1 \mathrm{wt} \%$, correspondingly (Jugo et al. 2005; Jugo 2009). Therefore, high oxygen fugacity can transform more sulfide to sulfate, which induces sulfur-undersaturated magmas and consequently allows assimilation of more sulfide. Copper is incompatible in sulfur-undersaturated magmas; when initial concentration of copper is high in oxidized magmas (Sun et al. 2013b, 2015a), conditions are favorable for copper mineralization. The oxygen fugacity in convergent-margin magmas is usually higher than in other geologic settings (e.g., intraplate setting), which is related to plate subduction and release of oxidized fluids (Brandon and Draper 1996; Parkinson and Arculus 1999; Sun et al. 2007a, b, 2010a). Therefore, the genesis of the high oxygen fugacity of the Shilu intrusion may be related to slab subduction. 

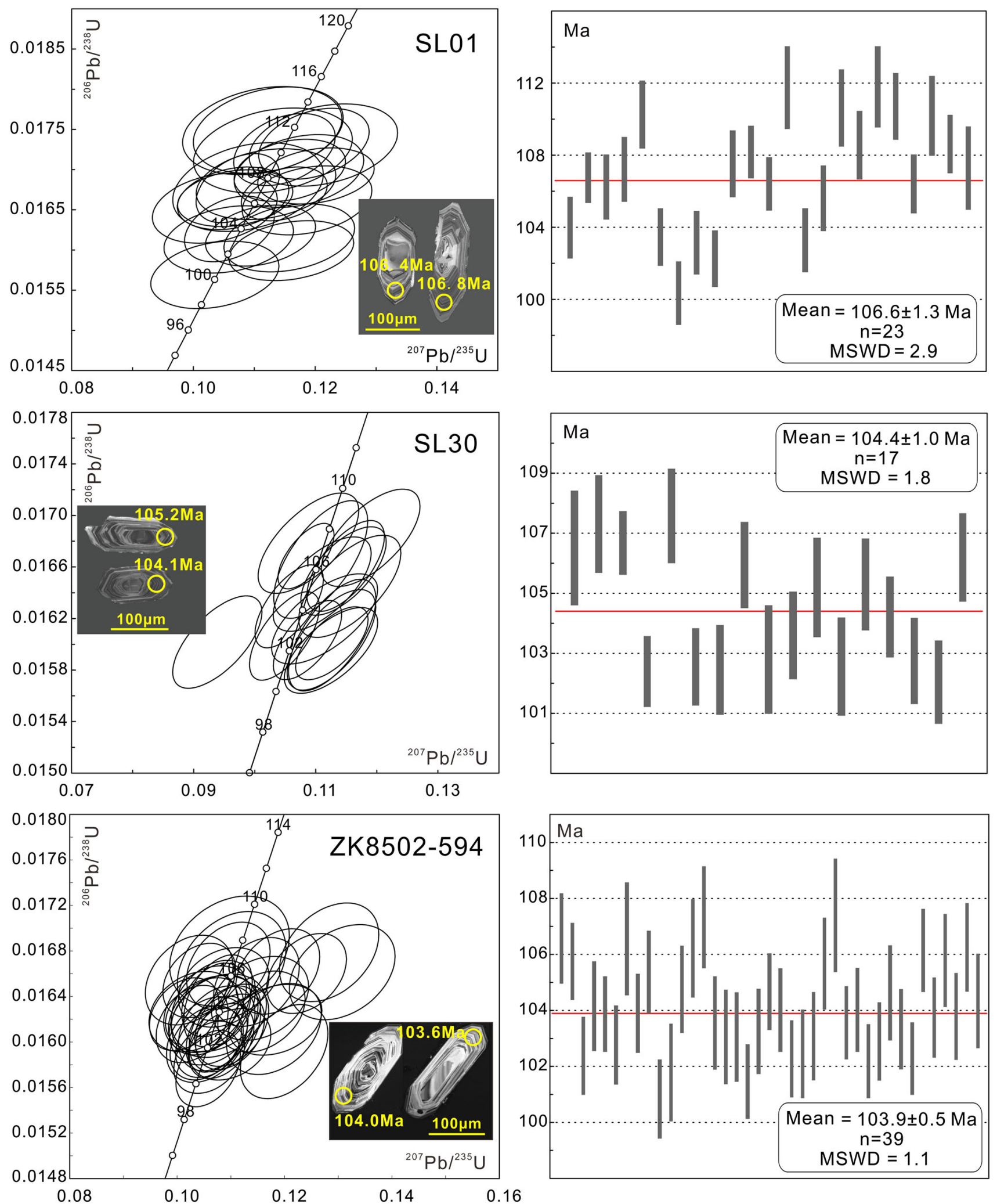

Fig. 5 Zircon U-Pb concordia and weighted mean age diagrams for the Shilu intrusion 


\subsection{Adakitic rock}

The term "adakite" was first proposed to describe magmas derived from partial melting of young, subducted oceanic slab (Defant and Drummond 1990). It was defined simply by its chemical composition. Typical adakite has $\mathrm{SiO}_{2}$ $\geq 56 \mathrm{wt} \%, \mathrm{Al}_{2} \mathrm{O}_{3} \geq 15 \mathrm{wt} \%, \mathrm{MgO}<3 \mathrm{wt} \%$, high $\mathrm{Sr}$ (usually $\geq 400 \mathrm{ppm}$ ), and low $\mathrm{Y}$ and $\mathrm{Yb}$ (generally $\leq 18$ and $1.9 \mathrm{ppm}$, respectively). Thus, adakite usually has high $\mathrm{Sr} / \mathrm{Y}$ and $\mathrm{La} / \mathrm{Yb}$ ratios. Shilu intrusive rocks measured here were consistent with the parameters of typical adakite, except that a few samples had slightly higher $\mathrm{Y}$ and $\mathrm{Yb}$ contents (Tables 1,4), which may have resulted from crustal contamination.

Because of the close genetic association between adakite and porphyry copper deposits, the rock has received great attention in recent years (Moyen 2009; Sun et al. 2010a, 2011, 2012a, 2013b; Hu et al. 2015). Given that adakite is defined by chemical characteristics, therefore, it may also be produced by partial melting of thickened lower continental crust (Chung et al. 2003; Wang et al. 2006). However, the average $\mathrm{Cu}$ concentration of lower continental crust is $\sim 26 \mathrm{ppm}$ (Rudnick and Gao 2003), and the mantle wedge may have copper contents lower than primitive mantle $(<30 \mathrm{ppm}$ ) (McDonough and Sun 1995;

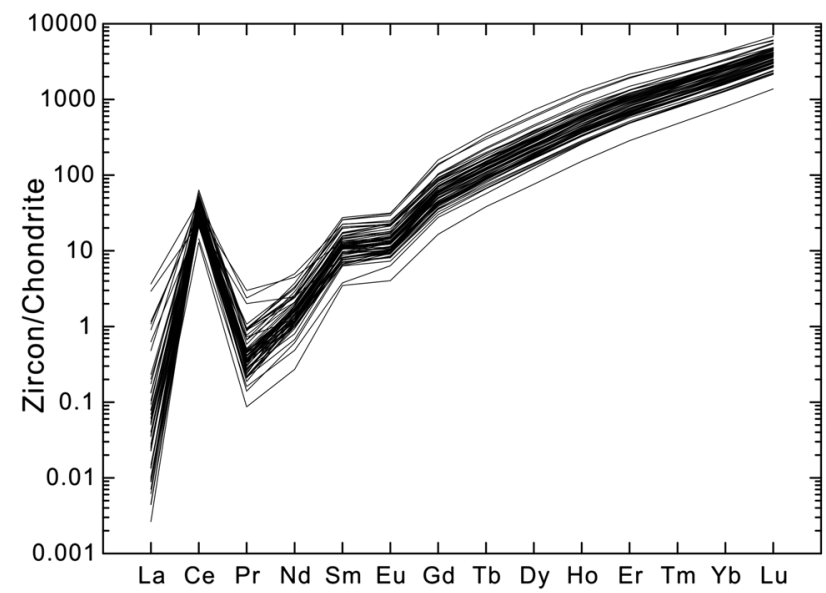

Fig. 6 Chondrite-normalized REE pattern diagram for zircons. Chondrite data are from Sun and McDonough (1989)
Sun et al. 2011); both have $\mathrm{Cu}$ concentrations much lower than that of oceanic crust $(\sim 100 \mathrm{ppm})$ (Sun et al. 2003, 2011). Therefore, adakitic magmas derived from

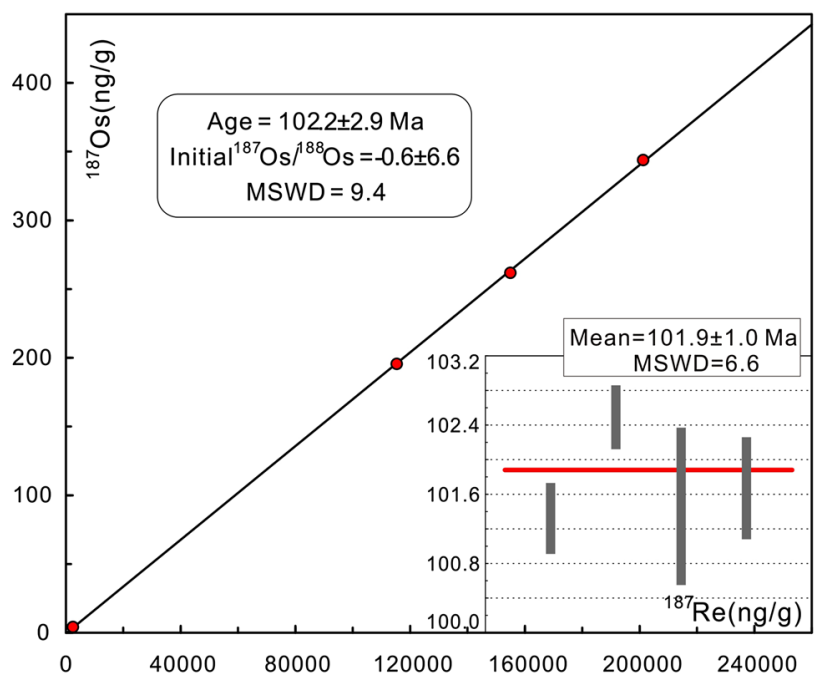

Fig. $7 \mathrm{Re}-\mathrm{Os}$ isochron and weighted mean age diagrams for the molybdenite samples from the Shilu deposit

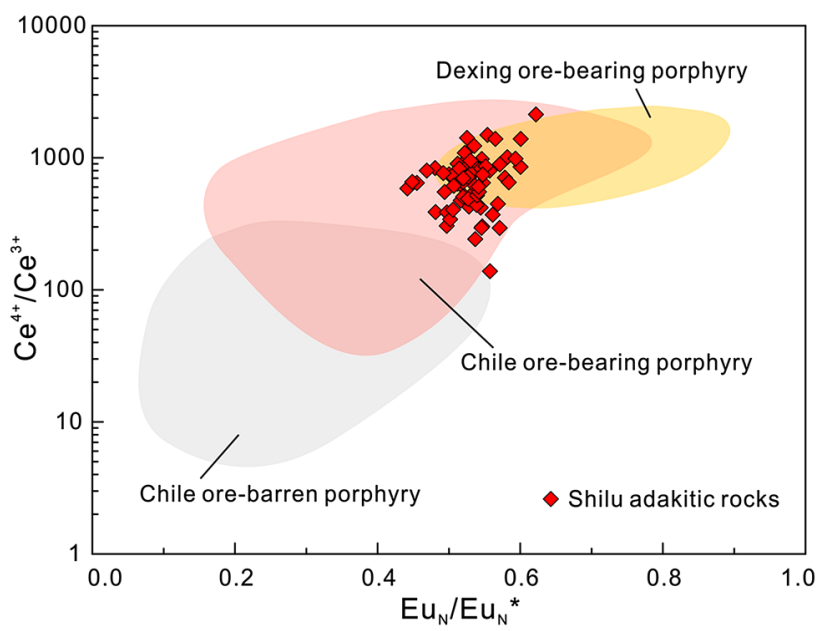

Fig. 8 Zircon $\mathrm{Eu}_{\mathrm{N}} / \mathrm{Eu}_{\mathrm{N}} *$ versus $\mathrm{Ce}^{4+} / \mathrm{Ce}^{3+}$ diagram. The fields of ore-bearing and ore-barren porphyry in Chile and ore-bearing porphyry in Dexing are modified from Zhang et al. (2013). The red rhombuses represent adakitic rocks from Shilu

Table $3 \mathrm{Re}$ and Os isotope data for the molybdenite samples from the Shilu $\mathrm{Cu}-\mathrm{Mo}$ deposit

\begin{tabular}{|c|c|c|c|c|c|c|c|c|c|}
\hline \multirow[t]{2}{*}{ Samples } & \multirow[t]{2}{*}{ Weight (g) } & \multicolumn{2}{|c|}{$\operatorname{Re}\left(\mu \mathrm{g} \cdot \mathrm{g}^{-1}\right)$} & \multicolumn{2}{|c|}{${ }^{187} \operatorname{Re}\left(\mathrm{ng} \cdot \mathrm{g}^{-1}\right)$} & \multicolumn{2}{|c|}{${ }^{187}$ Os (ng.g $\left.{ }^{-1}\right)$} & \multicolumn{2}{|l|}{ Age (Ma) } \\
\hline & & Content & $2 \sigma$ & Content & $2 \sigma$ & Content & $2 \sigma$ & Model age & $2 \sigma$ \\
\hline SL27-1 & 0.0150 & 246.5764 & 0.9658 & 154983 & 607 & 261.8365 & 0.2784 & 101.3 & 0.4 \\
\hline SL33 & 0.0150 & 320.1747 & 0.9074 & 201242 & 570 & 343.9037 & 0.7459 & 102.5 & 0.4 \\
\hline ZK4503-662 & 0.1028 & 3.8651 & 0.0171 & 2429.4 & 10.8 & 4.1097 & 0.0321 & 101.5 & 0.9 \\
\hline ZK4503-686 & 0.0147 & 183.5474 & 0.8640 & 115367 & 543 & 195.5667 & 0.6708 & 101.7 & 0.6 \\
\hline
\end{tabular}


oceanic crust should contain much higher $\mathrm{Cu}$ contents than those from the lower continental crust and are therefore favorable for $\mathrm{Cu}$ mineralization (Sun et al. 2011). The low oxygen fugacity of lower continental crust is another unfavorable factor for scavenging $\mathrm{Cu}$ from magma sources.

Different magma sources can also be effectively constrained using a multi-element diagram of $\mathrm{Sr} / \mathrm{Y}$ versus $(\mathrm{La} /$ $\mathrm{Yb})_{\mathrm{N}}$ (Ling et al. 2011; Liu et al. 2010; Sun et al. 2011). All our samples together with previous data plotted in the field of partial melting of subducted oceanic crust in Fig. 9. Combined with the above discussion, the most plausible mechanism for Shilu adakitic rocks and $\mathrm{Cu}-\mathrm{Mo}$ mineralization is the partial melting of subducted oceanic crust.

\subsection{Magma mixing and crustal contamination}

Previous researchers have shown that Shilu adakitic rocks have high radiogenic $\mathrm{Sr}$ and low $\varepsilon_{\mathrm{Nd}}(\mathrm{t})$ ( $\mathrm{Li}$ et al. 2001; Zheng et al. 2015). It is generally assumed that normal adakitic magmas derived from the partial melting of oceanic slab should have positive $\varepsilon_{\mathrm{Nd}}(\mathrm{t})$ and low radiogenic $\mathrm{Sr}$ (close to MORB in isotope composition), e.g., Cook adakite (Stern et al. 1984). However, because of assimilation of enriched mantle components as well as continental crust, the isotope composition is readily changed during magma ascent. In the two-component $\left({ }^{87} \mathrm{Sr} /{ }^{86} \mathrm{Sr}\right)_{\mathrm{i}}$ versus $\varepsilon_{\mathrm{Nd}}(\mathrm{t})$ mixing diagram, most of the Shilu samples fell near the field defined by the Lower Yangtze River Belt (LYRB) adakites and one sample in the field of the Dexing adakites (Fig. 10). Previous work has determined that the LYRB adakites were caused by ridge subduction, and these adakitic magmas mixed with type II enriched mantle (EMII) and were contaminated by crustal materials (Ling et al. 2009). The Dexing adakites were also derived from the partial melting of subducted oceanic crust and assimilated oceanic sediments or crustal materials (Zhang et al. 2013). In addition, the Nd isotope composition of the Shilu intrusion is notably higher than the Dabie adakites derived from the partial melting of lower continental crust (Ling et al. 2011). The South China Block is characterized by EMII (Li and Yang 2003) and the Shilu adakitic rocks are located on a mixing line between adakitic magmas and an

Table 4 Geochemical comparison of typical adakite and the Shilu intrusion

\begin{tabular}{lll}
\hline & Typical adakite & Shilu intrusion \\
\hline $\mathrm{SiO}_{2}$ & $\geq 56 \mathrm{wt} \%$ & $64.5-67.4 \mathrm{wt} \%$ \\
$\mathrm{Al}_{2} \mathrm{O}_{3}$ & $\geq 15 \mathrm{wt} \%$ & $15.4-16.6 \mathrm{wt} \%$ \\
$\mathrm{Sr}$ & $\geq 400 \mathrm{ppm}$ & $439-584 \mathrm{ppm}$ \\
$\mathrm{Y}$ & $\leq 18 \mathrm{ppm}$ & $15.9-22.3 \mathrm{ppm}$ \\
$\mathrm{Yb}$ & $\leq 1.9 \mathrm{ppm}$ & $1.55-2.30 \mathrm{ppm}$ \\
\hline
\end{tabular}

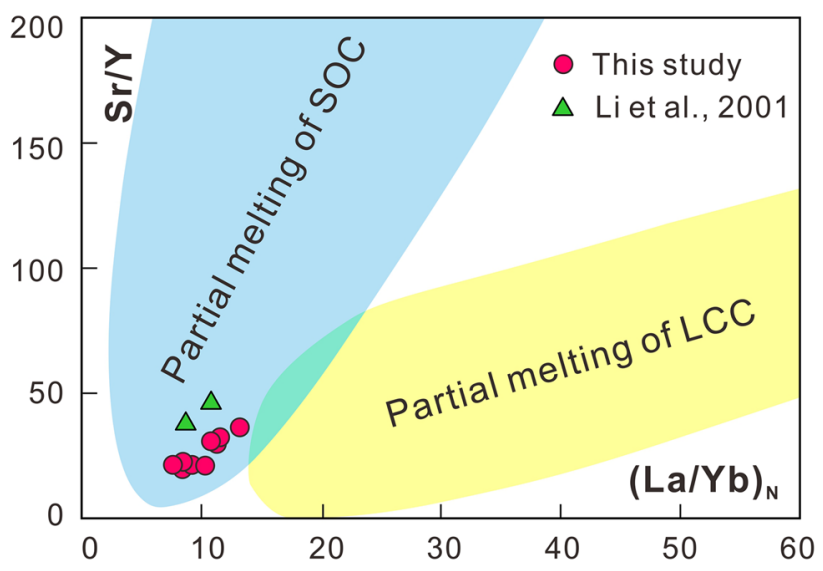

Fig. $9 \mathrm{Sr} / \mathrm{Y}$ versus $(\mathrm{La} / \mathrm{Yb})_{\mathrm{N}}$ diagram for the Shilu intrusion. The base map is modified after Ling et al. (2013). SOC subduction oceanic crust, LCC lower continental crust. $(\mathrm{La} / \mathrm{Yb})_{\mathrm{N}}$ ratios are chondritenormalized values, and chondrite date are from Sun and McDonough (1989)

EMII source, close to the mixing line between adakitic magmas and average continental crust. Therefore, Shilu adakitic rocks may be explained by the mixing of adakitic magmas with an EMII component contaminated by crustal materials or oceanic sediments. Zircon $\left({ }^{176} \mathrm{Hf} /{ }^{177} \mathrm{Hf}\right)_{i}$ isotope compositions of Shilu adakitic rocks are also higher than an oceanic crust source, indicating the contamination of crustal materials or oceanic sediments. The compositions of hornblende and biotite indicate that the Shilu intrusion is the product of crust-mantle mixed-source magma as well (Zheng et al. 2015). Crustal contamination is probably also responsible for the slightly higher $\mathrm{Y}$ and $\mathrm{Yb}$ contents than typical adakites in some samples. Magmatic contamination commonly occurs during magma ascent through assimilation. To some extent, assimilation is similar to partial melting, in that the wall rock is partially melted by intruding magma and the melt mixes into the magma. Both $\mathrm{Y}$ and $\mathrm{Yb}$ are moderately incompatible elements in the absence of garnet. The $\mathrm{Y}(20-21 \mathrm{ppm})$ and $\mathrm{Yb}$ (2.0-2.2 ppm) concentrations of the mid- and upper- continental crust (Rudnick and Gao 2003) are marginally higher than the discrimination line of adakite. Partial melting, however, produces magmas with $\mathrm{Y}$ and $\mathrm{Yb} \sim 3$ times higher than the source at $10 \%$ of partial melting. Therefore, crustal contamination elevates the $\mathrm{Y}$ and $\mathrm{Yb}$ contents.

Rhenium concentrations vary dramatically in molybdenites of different origin (Mao et al. 1999). The Re/Mo ratio of the continental crust $\left(2.5 \times 10^{-3}-2.5 \times 10^{-4}\right)$ (Rudnick and Gao 2003; Sun et al. 2003), however, corresponds to hundreds to thousands of ppm Re in molybdenite. Major fractionation between Re and Mo occurs during reduction. Organic-enriched sediments have low Re/Mo. Therefore, molybdenite formed in climax-type 


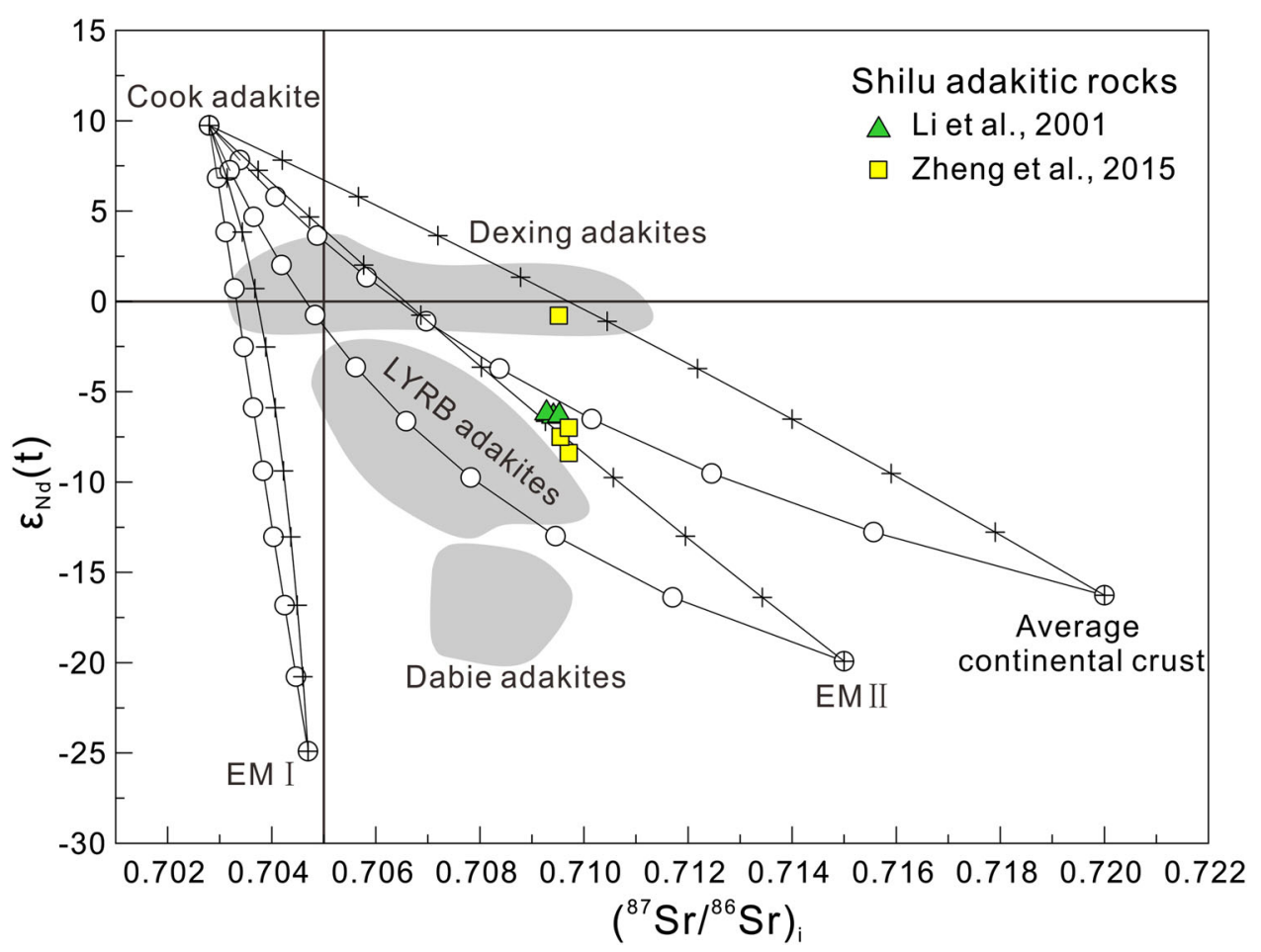

Fig. 10 Sr-Nd isotope mixing model for adakitic rocks in the Shilu deposit. Mixing simulation curves are modified after Ling et al. (2009). The Cook adakite is considered from the partial melting of oceanic plate and has a similar composition of Sr-Nd isotope with N-MORB (Kay et al. 1993; Stern et al. 1984); The compositions of EMI, EMII and average continental crust are from references (Guo et al. 2003; Hofmann 1997; Li and Yang 2003; Rudnick and Gao 2003). The mixing lines with circle symbols are for $\mathrm{Sr}=400 \mathrm{ppm}$, and the mixing lines with cross symbols are for $\mathrm{Sr}=1000 \mathrm{ppm}$. The fields of $\mathrm{Sr}-\mathrm{Nd}$ isotope compositions for Dexing adakites, LYRB adakites, and Dabie adakites are modified from Zhang et al. (2013), where LYRB Lower Yangtze River Belt

deposits has low Re, whereas that associated with subduction-related porphyry $\mathrm{Cu}$ deposits from South America have high Re (Sun et al. 2016a). In this study, the Re concentrations of molybdenite reached $\mathrm{n} \times 10^{-4}$ levels, similar to porphyry $\mathrm{Cu}$ deposits from South America (Sun et al. 2016a), with the exception of sample ZK4503-662 $\left(\mathrm{n} \times 10^{-6}\right)$. The high Re concentration in molybdenite is consistent with the slab melting model, whereas the low Re in sample ZK4503-662 is consistent with contamination of crustal materials with high organic concentrations.

Based on trace element analysis, we propose that the Shilu adakitic magmas were caused by the partial melting of subducted oceanic slab, which may have interacted with EMII and crustal materials during magma ascent.

\subsection{Geochronology}

Accurate isotopic age is crucial for understanding the relationships among magmatic rocks, mineralization, and tectonic events. Previous studies have obtained several ages for the Shilu intrusion. A zircon U-Pb evaporation age of $125 \mathrm{Ma}$ was obtained by the isotope geological age data assembly group in 1983; Zhao et al. (1985) determined an isochron age of $122 \pm 1$ Ma using mineral $\mathrm{Rb}-\mathrm{Sr}$ dating; and Yu et al. (1998) obtained an ${ }^{40} \mathrm{Ar}-{ }^{39} \mathrm{Ar}$ plateau age of 99-101 Ma through K-feldspar analysis. In recent years, LA-ICP-MS zircon U-Pb ages of $107 \pm 0.7,107.2 \pm 2.0$, $106.7 \pm 1.4$, and $104.1 \pm 2.0 \mathrm{Ma}$ have been obtained for granodiorites (Duan et al. 2013; Zheng et al. 2015). An oreforming age of $104.1 \pm 1.3 \mathrm{Ma}$ was obtained using molybdenite Re-Os dating (Zhao et al. 2012). The zircon evaporation age is much older than new results, likely because it is determined by ${ }^{207} \mathrm{~Pb} /{ }^{206} \mathrm{~Pb}$ and even a small amount of inherited $\mathrm{Pb}$ can perturb the analysis. It is also difficult to obtain precise mineral $\mathrm{Rb}-\mathrm{Sr}$ isochron ages at such young ages.

In this study, new zircon $\mathrm{U}-\mathrm{Pb}$ ages suggest that the Shilu intrusion formed between $106.6 \pm 1.3$ and $103.9 \pm 0.5 \mathrm{Ma}$. The oldest age is consistent with previously determined LA-ICP-MS zircon U-Pb ages (Duan et al. 2013; Zheng et al. 2015). The youngest zircon U-Pb age is younger than previously published ages. These results indicate multiple magmatic pulses. Consistently, previously published K-feldspar mineral ${ }^{40} \mathrm{Ar}-{ }^{39} \mathrm{Ar}$ plateau ages (99-101 Ma) are also slightly younger, which probably represent a thermal resetting event. Moreover, magmatic activity has been documented at around $100 \mathrm{Ma}$ in western Guangdong (Geng et al. 2006). For ore-forming 
age, molybdenite Re-Os dating defined an age of $102.2 \pm 2.9 \mathrm{Ma}$ in this study, which is consistent with previous findings (Zhao et al. 2012) and identical to the youngest zircon $\mathrm{U}-\mathrm{Pb}$ age within error, indicating a genetic relationship with $\mathrm{Cu}-\mathrm{Mo}$ mineralization.

\subsection{Tectonic setting}

As discussed above, Shilu adakitic rocks derived from the partial melting of subducted oceanic crust. The question then is: How could subducted oceanic crust reach Shilu? The South China Block was surrounded by the Pacific and the Neo-Tethys Oceans in the Cretaceous. Therefore, the most likely candidate is either the Pacific Plate or the NeoTethys Plate. In recent years, most researchers have argued that the subduction of the Pacific Plate may account for the formation of Cretaceous magmas and mineralization in South China.

Based on island chains, the Pacific Plate subducted southwestward beneath South China before 125 Ma (Sun et al. 2007a). It reached as far south as the Nanling Range at $\sim 160 \mathrm{Ma}$ and was immediately followed by slab rollback (Wang et al. 2011; Li et al. 2012; Sun et al. 2012b). Asthenosphere upwelling induced by slab rollback and phengite decomposition due to the elevated temperature released abundant fluorine and formed large-scale highly fractionated granites and $\mathrm{W}-\mathrm{Sn}$ mineralization in the Jurassic (Wang et al. 2011; Li et al. 2012). Therefore, the Pacific Plate before $125 \mathrm{Ma}$ was not responsible for adakitic rocks in the Yangchun district, south of the Nanling Range, and thus the partial melting of a Pacific slab cannot account for the Shilu adakitic rocks (Fig. 11). Furthermore, the Pacific Plate experienced a clockwise rotation of about $80^{\circ}$ at $\sim 125 \mathrm{Ma}$, changing the drift direction from southwest to northwest (Sun et al. 2007a, 2013a). As presented in previous studies, the ridge between the Pacific and Izanagi Plates subducted along the Lower Yangtze River belt at $\sim 140 \pm 10 \mathrm{Ma}$, forming extensive adakites and an important metallogenic belt (Ling et al. 2009; Sun et al. 2010a). The ridge then reached the Xuhuai arc at $\sim 130 \mathrm{Ma}$, forming Cretaceous adakites (Fig. 11) (Ling et al. 2013). The distance between the two adakite belts is about $500 \mathrm{~km}$, corresponding to a northward migration rate of $\sim 5 \mathrm{~cm} \cdot$ year $^{-1}$. The ridge migrated northward faster after $125 \mathrm{Ma}$ due to the reorientation and the northwestward subduction of the Pacific Plate. So, the ridge is likely located in the north of the Korean Peninsula. More than $2000 \mathrm{~km}$ separated the ridge and the position of the Shilu copper deposit at $\sim 104 \mathrm{Ma}$ (Fig. 11). Therefore, this Pacific ridge was not responsible for the Shilu adakitic rocks either.

The influence of the Neo-Tethys on the South China Block has received much less attention (Sun 2016) because its current trench is located to the south of the South China Sea, which is far away from the present position of the South China Block (Fig. 12). However, the position of the trench in the Cretaceous needs to be reconstructed because dramatic tectonic movements have occurred in Southeast Asia since that time. Considering the extrusion of the Indochina Block and the opening of the South China Sea (Tapponnier et al. 1982) and Borneo away from the South China Block (Ben-Avraham and Uyeda 1973), the trench should have been much closer to the South China Block at that time (Fig. 12).

The Neo-Tethys Plate started to subduct northward at $\sim 125 \mathrm{Ma}$ (Sun 2016). With normal plate subduction of the Neo-Tethys, however, it is difficult to not only reach Shilu but to get partial melting as well because it was still several hundred kilometers away from Shilu and would have been cold. However, if the Neo-Tethys ridge subducted at $\sim 104 \mathrm{Ma}$, that would be the best mechanism for Shilu adakitic rocks and copper mineralization (Fig. 13).

Ridge subduction is common along convergent margins, especially in South America. Many ridge subductions are associated with large-sized porphyry copper deposits, because of slab melting and high oxygen fugacities (Sun et al. 2010a). Oceanic crust closer to a spreading ridge is younger, hotter, and lighter, usually leading to low-angle subduction. Ridge subduction can extend far away from

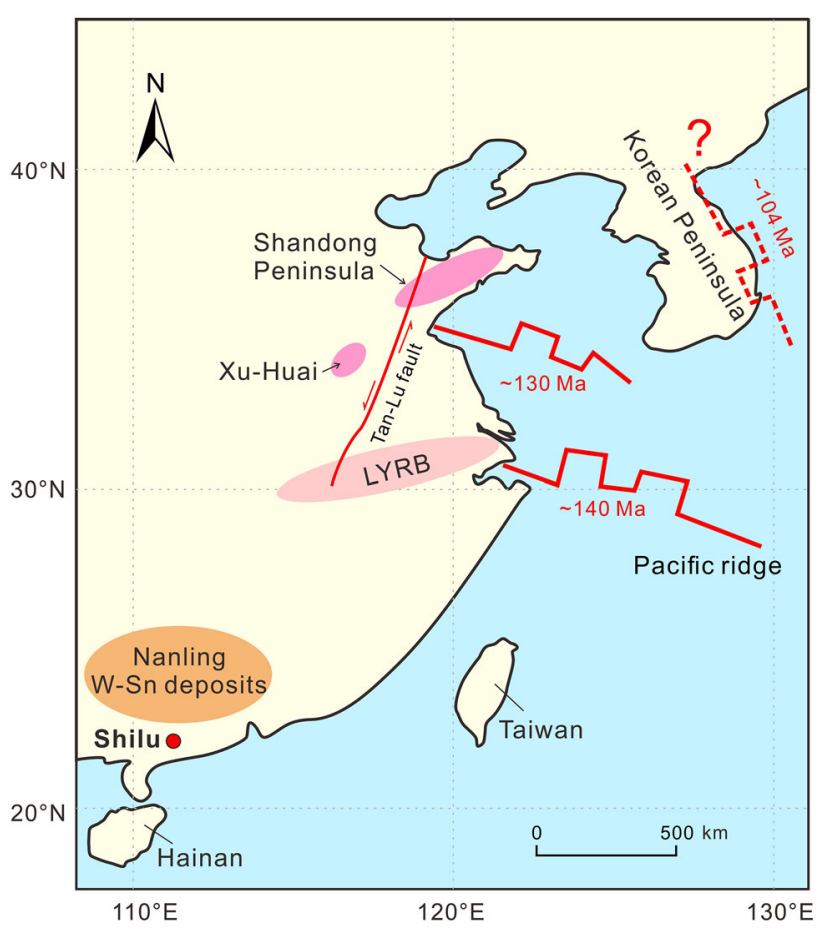

Fig. 11 Sketch map of magmatic belts in eastern China and different locations of the Pacific ridge at different times. The location of magmatic belts and the Pacific ridge are modified from Ling et al. (2013) 

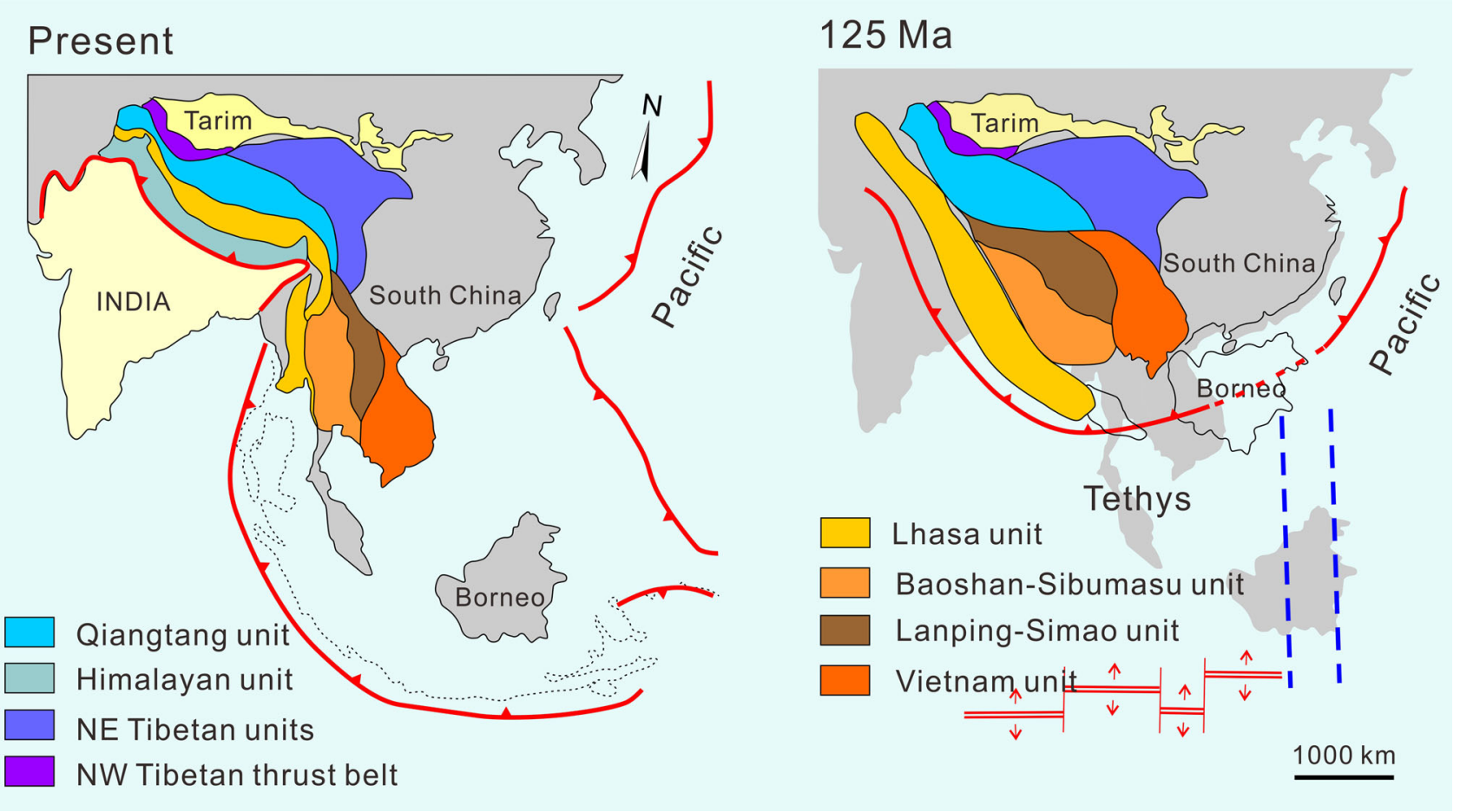

Fig. 12 Sketch map of present plates (Royden et al. 2008) and plates reconstruction at 125 Ma (based on Ben-Avraham and Uyeda 1973; Royden et al. 2008; Seton et al. 2012). Grey areas show the present location of blocks. Red lines are estimated positions of subduction zones. Blue lines show the boundary lines between the Pacific and the Tethys
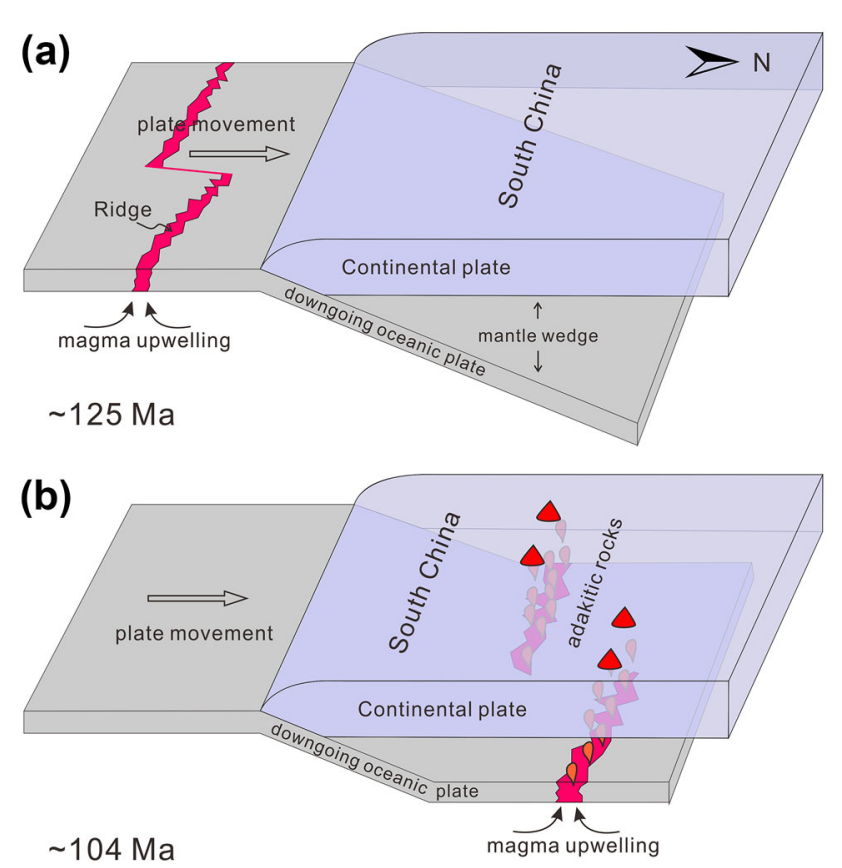

Fig. 13 Cartoon map illustrating the evolution history of the NeoTethys plate. a Neo-Tethys plate started to subduct northward at ca.125 Ma. b The Neo-Tethys ridge subducted beneath South China Block at $104 \mathrm{Ma}$. The ridge was parallel with subduction zone, which formed a magmatic line from Tibet Plateau to Canton in China subduction zones through flat subduction, e.g., over 1000 km (Coney and Reynolds 1977; Livaccari et al. 1981; Li and Li 2007; Espurt et al. 2008). The low fluorine and high chlorine of the Shilu intrusion also indicate shallow subduction, because chlorine in recycled oceanic crust has high mobility relative to fluorine in the early-stage of subduction (Lassiter et al. 2002). The Neo-Tethys ridge was parallel to the subduction zone, similar to the subduction of the northeast Pacific ridge in western North America (Cole and Basu 1995). Subduction of the NeoTethys ridge formed a magmatic line along the subduction zone from the Tibet Plateau to Canton in southern China (Fig. 13). A signature of Late Cretaceous ( 100 Ma) NeoTethys ridge subduction has been found in the Gangdese belt (Guan et al. 2010; Ye et al. 2015). In addition, Li et al. (2014) determined that South China tectonic evolution is characterized by the alternate appearance of compression and extension based on Cretaceous basin magmatism and paleo-tectonic stress field. There was a compression environment at around $110 \mathrm{Ma}$. To sum up, we propose that the eastern Neo-Tethys ridge was parallel to the subduction zone and subducted beneath the South China Block, and the partial melting of subducted oceanic crust formed the Shilu adakitic rocks associated with $\mathrm{Cu}-\mathrm{Mo}$ deposits. 


\section{Conclusions}

1. The Shilu intrusion has geochemical characteristics of adakitic rocks with high $\mathrm{Al}_{2} \mathrm{O}_{3}$ and $\mathrm{Sr}$, as well as high $\mathrm{Sr} / \mathrm{Y}$ and $\mathrm{La} / \mathrm{Yb}$ ratios, and low $\mathrm{Y}$ and $\mathrm{Yb}$ contents. It also has high oxygen fugacity as indicated by high zircon $\mathrm{Ce}^{4+} / \mathrm{Ce}^{3+}$ and $\mathrm{Eu}_{\mathrm{N}} / \mathrm{Eu}_{\mathrm{N}} *$ ratios.

2. Shilu adakitic magmas may have assimilated EMII and crustal materials during ascent and emplacement.

3. The Shilu intrusion formed between $106.6 \pm 1.3$ and $103.9 \pm 0.5 \mathrm{Ma}$, with multiple magmatic pulses. Our analysis returned a molybdenite $\mathrm{Re}-\mathrm{Os}$ isochron age of $102.2 \pm 2.9 \mathrm{Ma}$, which is identical to the youngest zircon $\mathrm{U}-\mathrm{Pb}$ age within error.

4. Shilu adakitic rocks formed by the partial melting of subducted oceanic crust at $\sim 107-103 \mathrm{Ma}$. The subduction of the Neo-Tethys ridge is the most likely source.

Acknowledgements This study was supported by the DREAM project of MOST China 2016YFC0600408, NSFC 91328204, 41421062, and China Geological Survey (12120114015801). GIGCAS contribution No. IS-000. We thank Congying Li for help with zircon U-Pb dating and Shengling Sun for assistance with molybdenite Re-Os dating. Zhixia Ouyang at Nonferrous Metals of Guangdong Province and Long Wang, Zhongxiang $\mathrm{He}$, and Lei Yang at Chengdu University of Technology are thanked for their help during field investigation.

Open Access This article is distributed under the terms of the Creative Commons Attribution 4.0 International License (http://crea tivecommons.org/licenses/by/4.0/), which permits unrestricted use, distribution, and reproduction in any medium, provided you give appropriate credit to the original author(s) and the source, provide a link to the Creative Commons license, and indicate if changes were made.

\section{References}

Ballard JR, Palin JM, Campbell IH (2002) Relative oxidation states of magmas inferred from $\mathrm{Ce}(\mathrm{IV}) / \mathrm{Ce}(\mathrm{III})$ in zircon: application to porphyry copper deposits of northern Chile. Contrib Mineral Petrol 144:347-364. doi:10.1007/s00410-002-0402-5

Ben-Avraham Z, Uyeda S (1973) The evolution of the China Basin and the Mesozoic paleogeography of Borneo. Earth Planet Sci Lett 18:365-376

BlichertToft J, Albarede F (1997) The Lu-Hf isotope geochemistry of chondrites and the evolution of the mantle-crust system. Earth Planet Sci Lett 148:243-258. doi:10.1016/s0012$821 \times(97) 00040-x$

Brandon AD, Draper DS (1996) Constraints on the origin of the oxidation state of mantle overlying subduction zones: an example from Simcoe, Washington, USA. Geochim Cosmochim Acta 60:1739-1749

Chen Z (1988) Geology-geochemistry characteristics of Xishan Granite Body in Yangchun, Guangdong. Acta Sci Nat Univ Sunyaatseni 1:1-12
Chung S-L, Liu D, Ji J, Chu M-F, Lee H-Y, Wen D-J, Lo C-H, Lee T-Y, Qian Q, Zhang Q (2003) Adakites from continental collision zones: melting of thickened lower crust beneath southern Tibet. Geology 31:1021-1024

Cole RB, Basu AR (1995) Nd-Sr isotopic geochemistry and tectonics of ridge subduction and middle Cenozoic volcanism in western California. Geol Soc Am Bull 107:167-179

Coney PJ, Reynolds SJ (1977) Cordilleran benioff zones. Nature 270:403-406

Defant MJ, Drummond MS (1990) Derivation of some modern arc magmas by melting of young subducted lithosphere. Nature 347:662-665

Duan R, Yang H, Zhang L, Liu C, Cai Y, Zhang F, Lin W, Mei Y (2013) U-Pb chronology of the granites in the Shilu Copper Ore deposit and its geological significance. Geotecton Metallog 37:754-764

Espurt N, Funiciello F, Martinod J, Guillaume B, Regard V, Faccenna C, Brusset S (2008) Flat subduction dynamics and deformation of the South American plate: insights from analog modeling. Tectonics 27:1-19

Geng H, Xu X, O'Reilly S, Zhao M, Sun T (2006) Cretaceous volcanic-intrusive magmatism in western Guangdong and its geological significance. Sci China Ser D 49:696-713

Gilder SA, Keller GR, Luo M, Goodell P (1991) Eastern Asia and the western Pacific timing and spatial distribution of rifting in China. Tectonophysics 197:225-243

Gilder SA, Gill J, Coe RS, Zhao X, Liu Z, Wang G, Yuan K, Liu W, Kuang G, Wu H (1996) Isotopic and paleomagnetic constraints on the Mesozoic tectonic evolution of south China. J Geophys Res Solid Earth (1978-2012) 101:16137-16154

Guan Q, Zhu D, Zhao Z, Zhang L, Liu M, Li X, Yu F, Mo X (2010) Late Cretaceous adakites in the eastern segment of the Gangdese Belt, southern Tibet: products of Neo-Tethyan ridge subduction? Acta Petrol Sin 26:2165-2179

Guo F, Fan W, Wang Y, Lin G (2003) Geochemistry of late Mesozoic mafic magmatism in west Shandong Province, eastern China: Characterizing the lost lithospheric mantle beneath the North China Block. Geochem J 37:63-77

Hofmann A (1997) Mantle geochemistry: the message from oceanic volcanism. Nature 385:219-229

Hu Y, Liu J, Ling M, Ding W, Liu Y, Re Zartman, Ma X, Liu D, Zhang C, Sun S, Zhang L, Wu K, Sun W (2015) The formation of Qulong adakites and their relationship with porphyry copper deposit: geochemical constraints. Lithos 220:60-80

Jugo PJ (2009) Sulfur content at sulfide saturation in oxidized magmas. Geology 37:415-418

Jugo PJ, Luth RW, Richards JP (2005) Experimental data on the speciation of sulfur as a function of oxygen fugacity in basaltic melts. Geochim Cosmochim Acta 69:497-503

Kay SM, Ramos V, Marquez M (1993) Evidence in Cerro Pampa volcanic rocks for slab-melting prior to ridge-trench collision in southern South America. J Geol 101:703-714

Lassiter J, Hauri E, Nikogosian I, Barsczus H (2002) Chlorinepotassium variations in melt inclusions from Raivavae and Rapa, Austral Islands: constraints on chlorine recycling in the mantle and evidence for brine-induced melting of oceanic crust. Earth Planet Sci Lett 202:525-540

Li X (2000) Cretaceous magmatism and lithospheric extension in Southeast China. J Asian Earth Sci 18:293-305

Li Z-X, Li X-H (2007) Formation of the 1300-km-wide intracontinental orogen and postorogenic magmatic province in Mesozoic South China: a flat-slab subduction model. Geology 35:179-182

Li S, Yang W (2003) Decoupling of surface and subsurface sutures in the Dabie orogen and a continent-collisional lithospheric-wedging model: $\mathrm{Sr}-\mathrm{Nd}-\mathrm{Pb}$ isotopic evidences of Mesozoic igneous rocks in eastern China. Chin Sci Bull 48:831-838 
Li X, Zhou H, Liu Y, Lee C, Chen Z, Yu J, Gui X (2000) Mesozoic shoshonitic intrusives in the Yangchun Basin, western Guangdong, and their tectonic significance: 1. Petrology and isotope geochronology. Geochimica 29:513-520

Li X, Zhou H, Liu Y, Lee C, Chen C, Yu J, Gui X (2001) Mesozoic shoshonitic intrusives in the Yangchun Basin, western Guangdong, and their tectonic significance: II. Trace elements and Sr$\mathrm{Nd}$ isotopes. Geochimica 30:57-65

Li X-H, Li Z-X, Li W-X, Liu Y, Yuan C, Wei G, Qi C (2007) U-Pb zircon, geochemical and $\mathrm{Sr}-\mathrm{Nd}-\mathrm{Hf}$ isotopic constraints on age and origin of Jurassic I-and A-type granites from central Guangdong, SE China: a major igneous event in response to foundering of a subducted flat-slab? Lithos 96:186-204

Li XH, Long WG, Li QL, Liu Y, Zheng YF, Yang YH, Chamberlain KR, Wan DF, Guo CH, Wang XC (2010) Penglai zircon megacrysts: a potential new working reference material for microbeam determination of $\mathrm{Hf}-\mathrm{O}$ isotopes and $\mathrm{U}-\mathrm{Pb}$ age. Geostand Geoanal Res 34:117-134

Li CY, Zhang H, Wang FY, Liu JQ, Sun YL, Hao XL, Li YL, Sun WD (2012) The formation of the Dabaoshan porphyry molybdenum deposit induced by slab rollback. Lithos 150:101-110. doi:10.1016/j.lithos.2012.04.001

Li J, Zhang Y, Dong S, Johnston ST (2014) Cretaceous tectonic evolution of South China: a preliminary synthesis. Earth Sci Rev 134:98-136

Liang H-Y, Campbell IH, Allen C, Sun W-D, Liu C-Q, Yu H-X, Xie Y-W, Zhang Y-Q (2006) Zircon $\mathrm{Ce}^{4+} / \mathrm{Ce}^{3+}$ ratios and ages for Yulong ore-bearing porphyries in eastern Tibet. Miner Depos 41:152-159

Lin J, Liu Y, Yang Y, Hu Z (2016) Calibration and correction of LAICP-MS and LA-MC-ICP-MS analyses for element contents and isotopic ratios. Solid Earth Sci 1:5-27

Ling MX, Wang FY, Ding X, Hu YH, Zhou JB, Zartman RE, Yang XY, Sun WD (2009) Cretaceous ridge subduction along the lower Yangtse River belt, eastern China. Econ Geol 104:303-321

Ling MX, Wang FY, Ding X, Zhou JB, Sun WD (2011) Different origins of adakites from the Dabie Mountains and the Lower Yangtze River Belt, eastern China: geochemical constraints. Int Geol Rev 53:727-740

Ling M-X, Li Y, Ding X, Teng F-Z, Yang X-Y, Fan W-M, Xu Y-G, Sun W (2013) Destruction of the North China Craton induced by ridge subductions. J Geol 121:197-213

Liu Y, Hu Z, Gao S, Günther D, Xu J, Gao C, Chen H (2008) In situ analysis of major and trace elements of anhydrous minerals by LA-ICP-MS without applying an internal standard. Chem Geol 257:34-43

Liu S-A, Li S, He Y, Huang F (2010) Geochemical contrasts between early Cretaceous ore-bearing and ore-barren high-Mg adakites in central-eastern China: implications for petrogenesis and $\mathrm{Cu}-\mathrm{Au}$ mineralization. Geochim Cosmochim Acta 74:7160-7178

Livaccari RF, Burke K, Şengör A (1981) Was the Laramide Orogeny related to subduction of an oceanic plateau? Nature 289:276-278

Ludwig KR (2003) User's manual for Isoplot 3.00: a geochronological toolkit for Microsoft Excel. Berkeley Geochronology Center, pp 1-70

Luo Nh (1988) The geochemical features and genesis of the Shilu Cu-Mo deposit, Yangchun, Guangdong. J Guilin College Geol 8:77-87

Mao J, Zhang Z, Zhang Z, Du A (1999) Re-Os isotopic dating of molybdenites in the Xiaoliugou W (Mo) deposit in the northern Qilian mountains and its geological significance. Geochim Cosmochim Acta 63:1815-1818

Mao J, Pirajno F, Cook N (2011) Mesozoic metallogeny in East China and corresponding geodynamic settings - an introduction to the special issue. Ore Geol Rev 43:1-7

McDonough WF, Sun S-S (1995) The composition of the Earth. Chemical geology 120:223-253
Mei Y, Yang H, Duan R, Zhang L, Cai Y (2013) Diagenetic and metallogenic geochronlogy study of the Xishan W-Sn ore deposit in Yangchun of Guangdong Province. Acta Geol Sin 87:1370-1375

Moyen J-F (2009) High Sr/Y and La/Yb ratios: the meaning of the "adakitic signature". Lithos 112:556-574

Mungall JE (2002) Roasting the mantle: slab melting and the genesis of major $\mathrm{Au}$ and $\mathrm{Au}$-rich $\mathrm{Cu}$ deposits. Geology 30:915-918

Parkinson IJ, Arculus RJ (1999) The redox state of subduction zones: insights from arc-peridotites. Chem Geol 160:409-423

Royden LH, Burchfiel BC, van der Hilst RD (2008) The geological evolution of the Tibetan Plateau. Science 321:1054-1058

Rudnick R, Gao S (2003) Composition of the continental crust. Treatise Geochem 3:1-64

Scherer E, Münker C, Mezger K (2001) Calibration of the lutetiumhafnium clock. Science 293:683-687

Seton M, Müller R, Zahirovic S, Gaina C, Torsvik T, Shephard G, Talsma A, Gurnis M, Turner M, Maus S (2012) Global continental and ocean basin reconstructions since $200 \mathrm{Ma}$. Earth Sci Rev 113:212-270

Sillitoe RH (2010) Porphyry copper systems. Econ Geol 105:3-41

Smoliar MI, Walker RJ, Morgan JW (1996) Re-Os ages of group IIA, IIIA, IVA, and IVB iron meteorites. Science 271:1099-1102

Stern C, Futa K, Muehlenbachs K (1984) Isotope and trace element data for orogenic andesites from the Austral Andes Andean magmatism. Springer, Berlin, pp 31-46

Sun W (2016) Initiation and evolution of the South China Sea: an overview. Acta Geochim 35:215-225

Sun S-S, McDonough W (1989) Chemical and isotopic systematics of oceanic basalts: implications for mantle composition and processes. Geol Soc Lond Spec Publ 42:313-345

Sun WD, Williams IS, Li SG (2002) Carboniferous and triassic eclogites in the western Dabie Mountains, east-central China: evidence for protracted convergence of the North and South China Blocks. J Metamorph Geol 20:873-886. doi:10.1046/j. 1525-1314.2002.00418.x

Sun W, Bennett V, Eggins S, Arculus R, Perfit M (2003) Rhenium systematics in submarine MORB and back-arc basin glasses: laser ablation ICP-MS results. Chem Geol 196:259-281

Sun WD, Ding X, Hu YH, Li XH (2007a) The golden transformation of the Cretaceous plate subduction in the west Pacific. Earth Planet Sci Lett 262:533-542. doi:10.1016/j.epsl.2007.08.021

Sun X, Tang Q, Sun W, Xu L, Zhai W, Liang J, Liang Y, Shen K, Zhang Z, Zhou B (2007b) Monazite, iron oxide and barite exsolutions in apatite aggregates from CCSD drillhole eclogites and their geological implications. Geochim Cosmochim Ac 71:2896-2905

Sun WD, Ling MX, Yang XY, Fan WM, Ding X, Liang HY (2010a) Ridge subduction and porphyry copper-gold mineralization: an overview. Sci China Earth Sci 53:475-484. doi:10.1007/s11430010-0024-0

Sun Y, Xu P, Li J, He K, Chu Z, Wang CY (2010b) A practical method for determination of molybdenite Re-Os age by inductively coupled plasma-mass spectrometry combined with Carius tube- $\mathrm{HNO}_{3}$ digestion. Analytical Methods 2:575-581

Sun W, Zhang H, Ling M-X, Ding X, Chung S-L, Zhou J, Yang X-Y, Fan W (2011) The genetic association of adakites and $\mathrm{Cu}-\mathrm{Au}$ ore deposits. Int Geol Rev 53:691-703

Sun W-D, Ling M-X, Chung S-L, Ding X, Yang X-Y, Liang H-Y, Fan W-M, Goldfarb R, Yin Q-Z (2012a) Geochemical constraints on adakites of different origins and copper mineralization. J Geol 120:105-120

Sun WD, Yang XY, Fan WM, Wu FY (2012b) Mesozoic large scale magmatism and mineralization in South China: preface. Lithos 150:1-5. doi:10.1016/j.lithos.2012.06.028

Sun W, Li S, Yang X, Ling M, Ding X, La Duan, Zhan M, Zhang H, Fan W (2013a) Large-scale gold mineralization in eastern China 
induced by an Early Cretaceous clockwise change in Pacific plate motions. Int Geol Rev 55:311-321

Sun WD, Liang HY, Ling MX, Zhan MZ, Ding X, Zhang H, Yang XY, Li YL, Ireland TR, Wei QR, Fan WM (2013b) The link between reduced porphyry copper deposits and oxidized magmas. Geochim Cosmochim Acta 103:263-275. doi:10.1016/j. gca.2012.10.054

Sun W, Huang R, Li H, Hu Y, Zhang C, Sun S, Zhang L, Ding X, Li C, Zartman RE (2015a) Porphyry deposits and oxidized magmas. Ore Geol Rev 65:97-131

Sun Y, Ren M, Xia X, Li C, Sun W (2015b) Determination of Os by isotope dilution-inductively coupled plasma-mass spectrometry with the combination of laser ablation to introduce chemically separated geological samples. Spectrochim Acta Part B 113:22-29

Sun W, Li C, Hao X, Ling M, Ireland T, Ding X, Fan W (2016a) Oceanic anoxic events, subduction style and molybdenum mineralization. Solid Earth Sci 1:64-73

Sun W, Lin C-T, Zhang C, Hu Y, Ling M-X, Ding X, Li C-Y, Li H, Wu K, Fan W (2016b) Erratum to: Initiation and evolution of the South China Sea: an overview. Acta Geochim 35:1

Tapponnier P, Peltzer G, Le Dain A, Armijo R, Cobbold P (1982) Propagating extrusion tectonics in Asia: new insights from simple experiments with plasticine. Geology 10:611-616

Wang Q, Wyman DA, Xu J-F, Zhao Z-H, Jian P, Xiong X-L, Bao Z-W, Li C-F, Bai Z-H (2006) Petrogenesis of Cretaceous adakitic and shoshonitic igneous rocks in the Luzong area, Anhui Province (eastern China): implications for geodynamics and $\mathrm{Cu}-$ Au mineralization. Lithos 89:424-446

Wang FY, Ling MX, Ding X, Hu YH, Zhou JB, Yang XY, Liang HY, Fan WM, Sun WD (2011) Mesozoic large magmatic events and mineralization in SE China: oblique subduction of the Pacific plate. Int Geol Rev 53:704-726. doi:10.1080/00206814.2010. 503736

Wu J, Zeng X (2011) Geological characteristics of Nanshan Bengkeng-shiwu lead-zinc-copper-tin deposit in Yangchun City, Guangdong Province. Resour Environ Eng 25:201-208

$\mathrm{Wu}$ Y, Zheng Y (2004) Genesis of zircon and its constraints on interpretation of U-Pb age. Chin Sci Bull 49:1554-1569

Wu F-Y, Yang Y-H, Xie L-W, Yang J-H, Xu P (2006) Hf isotopic compositions of the standard zircons and baddeleyites used in U$\mathrm{Pb}$ geochronology. Chem Geol 234:105-126
Ye L, Zhao Z, Liu D, Zhu D, Dong G, Mo X, Hu Z, Liu Y (2015) Late Cretaceous diabase and granite dike in Namling, Tibet: petrogenesis and implications for extension. Acta Petrol Sin 31:1298-1312

Yu J, Gui X, Li P, Yuan C (1998) Isotope and trace element geochemistry of granitoid plutons in Yangchun basin, Guangdong. Guangdong Geol 13:1-10

Zhang X (2008) The discussion of the genetic type and mineralization of Shilu $\mathrm{Cu}$ (Mo) deposit in Yangchun. Chem Eng Equip 10:62-65

Zhang X, Zhang H (2012) The distribution of Shilu Cu-Mo orebodies in Yangchun, Guangdong. China Sci Technol Inf 9:37-44

Zhang H, Ling M-X, Liu Y-L, Tu X-L, Wang F-Y, Li C-Y, Liang H-Y, Yang X-Y, Arndt NT, Sun W-D (2013) High oxygen fugacity and slab melting linked to $\mathrm{Cu}$ mineralization: evidence from Dexing porphyry copper deposits, southeastern China. J Geol 121:289-305

Zhao Z, Ma D, Lin H, Zhang X (1985) Rubidium-strontium and oxygen isotopic composition of the two types of granitoids in Yangchun area, Guangdong province and discussion on their origin. Bull Yichang Inst Geol Miner Resour CAGS 10:89-97

Zhao H, Zheng W, Yu Z, Hu Y, Tian Y (2012) Re-Os dating of molybdenite from the Shilu $\mathrm{Cu}$ (Mo) deposit in western Guangdong Province and its geological implications. Geol China 39:1604-1613

Zheng W, Chen M, Xu L, Zhao H, Ling S, Wu Y, Hu Y, Tian Y, Wu $\mathrm{X}$ (2013) $\mathrm{Rb}-\mathrm{Sr}$ isochron age of Tiantang $\mathrm{Cu}-\mathrm{Pb}-\mathrm{Zn}$ polymetallic deposit in Guangdong Province and its geological significance. Miner Depos 32:259-272

Zheng W, Mao J, Pirajno F, Zhao H, Zhao C, Mao Z, Wang Y (2015) Geochronology and geochemistry of the Shilu $\mathrm{Cu}-\mathrm{Mo}$ deposit in the Yunkai area, Guangdong Province, South China and its implication. Ore Geol Rev 67:382-398

Zhou X, Li W (2000) Origin of Late Mesozoic igneous rocks in Southeastern China: implications for lithosphere subduction and underplating of mafic magmas. Tectonophysics 326:269-287

Zhou X, Sun T, Shen W, Shu L, Niu Y (2006) Petrogenesis of Mesozoic granitoids and volcanic rocks in South China: a response to tectonic evolution. Episodes 29:26-33 